\title{
A MID-INFRARED IMAGING SURVEY OF SUBMILLIMETER-SELECTED GALAXIES WITH THE SPITZER SPACE TELESCOPE
}

\author{
Laura J. Hainline ${ }^{1,8}$, A. W. Blain ${ }^{1}$, Ian Smail ${ }^{2}$, D. T. Frayer ${ }^{3}$, S. C. Chapman ${ }^{4}$, R. J. Ivison ${ }^{5,6}$, And D. M. Alexander ${ }^{7}$ \\ ${ }^{1}$ Department of Astronomy, California Institute of Technology, Mail Code 105-24, Pasadena, CA 91125, USA; 1jh@astro.caltech.edu, ljh@astro.umd.edu \\ 2 Institute for Computational Cosmology, Durham University, South Road, Durham DH1 3LE, UK \\ ${ }^{3}$ Infrared Processing and Analysis Center, California Institute of Technology, Mail Code 100-22, Pasadena, CA 91125, USA \\ ${ }^{4}$ Institute for Astronomy, University of Cambridge, Madingley Road, Cambridge CB3 OHA, UK \\ ${ }^{5}$ Institute for Astronomy, University of Edinburgh, Blackford Hill, Edinburgh EH9 3HJ, UK \\ ${ }^{6}$ UK Astronomy Technology Centre, Royal Observatory, Blackford Hill, Edinburgh EH9 3HJ, UK \\ ${ }^{7}$ Department of Physics, Durham University, Durham DH1 3LE, UK \\ Received 2008 August 26; accepted 2009 May 11; published 2009 June 24
}

\begin{abstract}
We present Spitzer-IRAC and MIPS mid-IR observations of a sample of 73 radio-detected submillimeter-selected galaxies (SMGs) with spectroscopic redshifts, the largest such sample published to date. From our data, we find that IRAC colors of SMGs are much more uniform as compared with rest-frame UV and optical colors, and $z>1.5$ SMGs tend to be redder in their mid-IR colors than both field galaxies and lower- $z$ SMGs. However, the IRAC colors of the SMGs overlap those of field galaxies sufficiently that color-magnitude and color-color selection criteria suggested in the literature to identify SMG counterparts produce ambiguous counterparts within an $8^{\prime \prime}$ radius in $20 \%-35 \%$ of cases. We use a rest-frame $J-H$ versus $H-K$ color-color diagram and a $S_{24} / S_{8.0}$ versus $S_{8.0} / S_{4.5}$ color-color diagram to determine that $13 \%-19 \%$ of our sample are likely to contain active galactic nuclei which dominate their mid-IR emission. We observe in the rest-frame JHK colors of our sample that the rest-frame near-IR emission of SMGs does not resemble that of the compact nuclear starburst observed in local ultraluminous IR galaxies and is consistent with more widely distributed star formation. We take advantage of the fact that many high- $z$ galaxy populations selected at different wavelengths are detected by Spitzer to carry out a brief comparison of mid-IR properties of SMGs to UV-selected high- $z$ galaxies, $24 \mu \mathrm{m}$-selected galaxies, and high- $z$ radio galaxies, and find that SMGs have mid-IR fluxes and colors which are consistent with being more massive and more reddened than UV-selected galaxies, while the IRAC colors of SMGs are most similar to powerful high- $z$ radio galaxies.
\end{abstract}

Key words: galaxies: evolution - galaxies: formation - galaxies: high-redshift - infrared: galaxies

Online-only material: color figures, machine-readable tables

\section{INTRODUCTION}

Through study of the far-IR/submillimeter background and the brightest galaxies resolved from it, we know that a population of infrared (IR)-luminous galaxies contributed significantly to the star formation history of the universe at high redshifts. Since their discovery over a decade ago in surveys with the Submillimeter Common-User Bolometer Array (SCUBA; Holland et al. 1999) instrument on the $15 \mathrm{~m}$ James Clerk Maxwell Submillimeter Telescope (JCMT; e.g., Smail et al. 1997; Hughes et al. 1998; Barger et al. 1998), tremendous progress has been made in revealing the properties of the brightest galaxies resolved from the submillimeter (submm) background, the highly luminous population of submm-selected galaxies (SMGs). Through extensive multiwavelength followup observations it has been shown that these galaxies tend to lie at high redshift (median $z=2.2$; Chapman et al. 2005), and are massive (e.g., Swinbank et al. 2004; Borys et al. 2005; Tacconi et al. 2008) and gas-rich (e.g., Neri et al. 2003; Greve et al. 2005; Tacconi et al. 2006). Despite their large bolometric luminosity $\left(L_{\mathrm{IR}}>10^{12} L_{\odot}\right.$; Chapman et al. 2005; Kovács et al. 2006; Pope et al. 2006), SMGs are known to be typically very faint at optical wavelengths and near-IR wavelengths (e.g., Smail et al. 1998, 2004; Ivison et al. 2002; Chapman et al. 2005), which is attributed to strong dust obscuration at rest-frame UV and op-

\footnotetext{
8 Current address: Department of Astronomy, University of Maryland, College Park, MD 20742, USA.
}

tical wavelengths. The tremendous star formation rates implied by the IR luminosities of SMGs (SFR $\sim 10^{3} M_{\odot} \mathrm{yr}^{-1}$ ), exceeding that of local Ultra-Luminous IR Galaxies (ULIRGs), have led to speculation that SMGs represent the formation phase of the most massive spheroids (e.g., Lilly et al. 1999; Smail et al. 2002). A large contribution to the bolometric output of SMGs from accretion onto a central supermassive black hole could mimic a high star formation rate, but deep X-ray observations from the Chandra X-ray Observatory and mid-IR spectra from the Spitzer Space Telescope suggest that while it is likely that most SMGs host an active galactic nucleus (AGN), only $10 \%-$ $20 \%$ of the far-IR luminosity of SMGs is powered by obscured AGNs unless the nuclei are Compton-thick (Alexander et al. 2003, 2005a; Menéndez-Delmestre et al. 2009).

With characterization of the bright $\left(S_{850 \mu \mathrm{m}}>5 \mathrm{mJy}\right) \mathrm{SMG}$ population underway, the challenge now is to determine what role they played in the overall star formation rate density at high redshift and also in galaxy evolution. If the IR luminosities of SMGs are powered by star formation, a higher star formation rate density is implied than that determined from optically selected galaxies. The increase in star formation rate density is difficult to reconcile with standard semi-analytic models of galaxy formation (Baugh et al. 2005, 2007; Swinbank et al. 2008), and suggest that massive galaxies formed the bulk of their stellar populations very quickly. On the other hand, if much of the luminosity of the submm sources derives from accretion onto a central, supermassive black hole, then they may represent important phases in supermassive black hole 
formation and growth and the evolution of active galaxies. Understanding the frequency and nature of luminous submm sources is thus important for determining the assembly history of massive galaxies.

Determining the contribution of SMGs to the cosmic mass assembly history will require the assembly of large samples of SMGs; however, the follow-up observations of such samples of faint galaxies, from identifying counterparts at optical/nearIR/radio wavelengths to determining spectroscopic redshifts and AGN contribution to the bolometric luminosity, will remain resource-intensive (see, e.g., Ivison et al. 2002; Chapman et al. 2005; Pope et al. 2006). In preparation for the next wave of large submm surveys with forthcoming SCUBA-2 instrument at JCMT, the Herschel Space Telescope, and eventually the Cornell-Caltech Atacama Telescope (CCAT), which will be important in studies of the role of SMGs in the cosmic star formation history, it will be extremely useful to find less observationally intensive methods of finding SMG counterparts, estimating/determining their redshifts, and identifying AGNdominated sources, which may permit us to avoid the more time-consuming parts of the follow-up. While attempts to do so with optical-wavelength data have not been very fruitful (e.g., Smail et al. 1998; Barger et al. 1999b), in the nearand mid-IR the effects of dust obscuration are reduced and very hot dust heated by an AGN appear, which may help with redshift determination and separation of powerful AGNs from starbursts. Spitzer permits determination of the rest-frame near and mid-IR properties of SMGs; several authors (e.g., Egami et al. 2004; Pope et al. 2006; Dye et al. 2008) have already shown that SMGs can be detected and studied with Spitzer. Yet, none so far have analyzed a large, representative sample with spectroscopic redshifts to examine the different effects of redshift, AGN contribution, and stellar contribution separately.

Another key component in understanding the role of SMGs in the context of galaxy evolution is to understand the relationships between them and the various other populations of high-redshift galaxies, e.g., UV-selected galaxies (Lyman break galaxies, BX/ BM galaxies; Steidel et al. 2003), optically selected galaxies (e.g., Balmer/4000 A-Break Galaxies, BzKs; Franx et al. 2003; Daddi et al. 2004), and radio-selected galaxies. Whether the different populations selected at different wavelengths represent an evolutionary sequence, a mass sequence, or products of different environments remains an open question. Comparing the different populations directly has so far not been a simple task, since current instrumentation does not permit the easy detection of some populations at all wavelengths (e.g., the ability to detect Lyman break galaxies at optical wavelengths but not at submm or radio wavelengths unless gravitationally lensed), and spectroscopic redshifts are needed to best interpret comparisons of different galaxy populations. However, the Spitzer bands present a region of overlap for all of the high- $z$ populations, as a variety of studies (e.g., Reddy et al. 2006; Papovich et al. 2006; Seymour et al. 2007) indicate that a large fraction of the different high- $z$ populations are well detected in the Spitzer bands. Thus, Spitzer data provide the best opportunity, so far, to compare and contrast SMGs with high- $z$ galaxies selected at other wavelengths.

In this paper, we describe an observational survey with Spitzer-IRAC and MIPS of the radio-detected SMG sample of Chapman et al. (2005), which is the largest sample of SMGs with spectroscopic redshifts, and characterize the rest-frame near-IR properties for this representative sample of SMGs. We attempt to address some fundamental questions regarding SMGs. First, we would like to know if SMGs have distinctive rest-frame nearIR colors, which will assist in the identification of counterparts to known SMGs and highlight potential SMGs lurking in the rich Spitzer data archive. Second, we are interested in looking for any redshift-dependent trends in the near- and mid-IR data which may be exploited to determine the redshifts of SMGs without resorting to spectroscopy. Third, we want to know if we can discriminate SMGs with energetically dominant AGNs from those dominated by starlight based on their near- and mid-IR properties. Finally, we wish to compare Spitzer properties and colors of SMGs with those of high-redshift galaxies selected at other wavelengths to shed some light on the relationships among them. In L. J. Hainline et al. (2009a, 2009b, in preparation; hereafter Papers II and III), we use the results of our Spitzer observations of SMGs described here to derive the total IR luminosities, stellar masses, and constrain stellar population characteristics of these galaxies via their rest-frame UV/optical through far-IR spectral energy distributions (SEDs). All of these analyses require accurate spectroscopic redshifts for proper characterization.

We begin in Section 2 by describing our SMG sample and our IRAC and MIPS $24 \mu \mathrm{m}$ and $70 \mu \mathrm{m}$ observations. In Section 3, we present the IRAC and MIPS properties for the sample. We then discuss the near-IR fluxes and colors of SMGs in relation to their redshifts, deep field galaxy samples, AGN content, and other populations of high- $z$ galaxies in Section 4.

\section{OBSERVATIONS}

\subsection{The SMG Sample}

In our survey, we image the fields targeted in the SMG spectroscopic survey of Chapman et al. (2005, C05 hereafter). The sample consists of 73 SCUBA galaxies with spectroscopic redshifts in seven different "blank" fields on the sky: CFRS-03 (cf., Eales et al. 1999; Webb et al. 2003), Lockman Hole East (cf. Scott et al. 2002; Ivison et al. 2002), Hubble Deep FieldNorth(HDF-N)/GOODS-N (cf., Barger et al. 2000), SSA 13 (cf., Barger et al. 2001), CFRS-14 (cf., Eales et al. 1999, 2000), ELAIS-N2 (cf., Scott et al. 2002; Ivison et al. 2002), and SSA 22 (cf., Barger et al. 1999a). The parent sample of $\sim 100 S_{850} \gtrsim 4 \mathrm{mJy}$ SMGs from which the spectroscopic redshift sample derives were chosen without regard for optical brightness. None of the galaxies in the sample are thought to be strongly lensed. C05 find a median of $z=2.2$ for the redshift distribution of our sample, $S_{1.4}=78 \pm 106 \mu \mathrm{Jy}$ for the $1.4 \mathrm{GHz}$ flux distribution, $S_{850}=5.7 \pm 3.0 \mathrm{mJy}$ for the $850 \mu \mathrm{m}$ flux distribution, and median $R$-magnitude $R_{\mathrm{AB}}=24.6 \pm 1.7$. For $25 \%$ of the sample, C05 find spectral signatures of AGN in the rest-frame UV spectra, while $40 \%$ have spectral characteristics of star-forming galaxies; the rest of the UV spectra are difficult to classify.

To obtain accurate positions for submm sources detected by SCUBA for optical spectroscopy, C05 required that the submm sources be detected in very deep ( $1 \sigma$ noise $\sim 5-11 \mu \mathrm{Jy})$, highresolution $\left(\sim 1^{\prime \prime}\right)$ Very Large Array (VLA) maps at $1.4 \mathrm{GHz}$. Since the far-IR emission from galaxies strongly correlates with radio continuum emission at both low and high redshift (e.g., Condon 1992; Appleton et al. 2004; Kovács et al. 2006), the radio emission from galaxies traces the same regions of recent massive star formation powering the galaxy's far-IR emission. High spatial resolution radio maps are thus complementary to the submm observations and pinpoint the sources of high- $z$ farIR emission observed in the submm, corroborated by targeted 
SCUBA photometry of faint radio sources (Barger et al. 2000; Chapman et al. 2001, 2002). The utility of high-resolution interferometric radio continuum maps was recognized early in the study of SMGs (e.g., Ivison et al. 1998) and found to be efficient at identifying precise positions for large numbers of submm sources and IR-luminous galaxies at high redshift (e.g., Smail et al. 2000; Barger et al. 2000) due to the far-IRradio correlation and the fact that the faint $1.4 \mathrm{GHz}$ source density on the sky is orders of magnitude lower than at optical wavelengths.

The association of the SMGs in the C05 sample with radio counterparts, for which redshifts were determined, was carried out through a probability analysis similar to that presented in Ivison et al. (2002). For a radio source to be selected as the counterpart to the submm source, it was required to have a probability $P$ that it was not associated with the submm emission of less than 0.05. In addition, one quarter of the C05 SMG sample are radio sources which were targeted for SCUBA photometry and have measurements taken individually with SCUBA in the "PHOT" mode; thus, there is less ambiguity in the association of the radio source with the submm source. Nine SMGs in the C05 sample (6\% of the full submm sample and $12 \%$ of the radio-detected sample) have two possible statistically robust radio identifications; we are confident in these cases that the correct radio counterpart has been selected from followup observations of the particular galaxies. Seven of the nine SMGs with multiple radio counterparts have been observed with the Infrared Spectrograph (IRS) instrument on Spitzer; the IRS spectra confirm that the dominant mid-IR source (and presumably far-IR source) lies at the redshift of the radio counterpart selected as the SMG. Of the remaining SMGs with multiple radio counterparts, one has been identified as a merging, far-IR luminous system at the redshift of the radio counterpart selected by $\mathrm{C} 05$ through high spatial resolution CO observations (Tacconi et al. 2008), while for the other the spectroscopic redshifts of both radio counterparts are available $(z=2.37$ and $z=0.41)$ and the radio luminosity of the higher redshift radio counterpart implies that it is more likely to be a far-IR luminous galaxy.

At least $50 \%$ of the redshifts have been verified through independent spectra taken at one or more of near-IR (e.g., $\mathrm{H} \alpha$ identification, Swinbank et al. 2004; Takata et al. 2006), midIR (e.g., Menéndez-Delmestre et al. 2007, 2009; Valiante et al. 2007; Pope et al. 2008), or millimeter (CO rotational lines, e.g., Greve et al. 2005; Tacconi et al. 2006, 2008) wavelengths; 35 SMGs in our sample have been observed with IRS on Spitzer. For seven SMGs in our sample, alternate redshifts than found in C05 have been proposed by Pope et al. (2008) and Menéndez-Delmestre et al. (2009), based on polycyclic aromatic hydrocarbon (PAH) emission lines in their mid-IR spectra and deep optical spectra; we adopt the revised redshifts here. The difference between the IRS redshift and the C05 redshift generally lies in the range 5\%-20\% and does not result in significant changes to derived luminosities (see details in Menéndez-Delmestre et al. 2009). These seven SMGs, which represent $20 \%$ of the sample observed with IRS, generally had noisy and/or ambiguous optical spectra in $\mathrm{C} 05$; in none of the cases were the radio counterparts found to be the incorrect identification. The redshifts of an additional 10 SMGs in the C05 may be regarded with some suspicion, as the radio counterparts for these SMGs are offset from the spectroscopically identified galaxy. However, half of these SMGs have had their redshifts confirmed through IRS spectra taken at the position of the radio source, lending increased credibility to the redshifts of SMGs which remain unconfirmed.

The spectroscopic SMG sample of C05 is the most complete and representative sample of this population and has been invaluable to the characterization of SMGs at all wavelengths, especially for studies of their luminosity, their relation to other high-redshift populations, and the role of SMGs in the stellar mass assembly history of the universe. However, it does suffer from significant selection biases. The spectroscopic redshifts are slightly biased toward brighter optical galaxies, although the radio and submm flux distributions of the galaxies with successful redshifts are not significantly different from those galaxies observed for which redshifts could not be determined. The other notable selection biases are those resulting from the flux limits of the submm and radio surveys $(\sim 4 \mathrm{mJy}$ and $\sim 30 \mu \mathrm{Jy}$, respectively). Around $65 \%-70 \%$ of bright SMGs have been detected in the radio (e.g., Ivison et al. 2002; Borys et al. 2004) to such flux levels, though the remaining $~ 30 \%$ span the same range in $S_{850}$ as the radio-detected sample. Thus, in requiring a radio detection, $\mathrm{C} 05$ limit their study to a subsample of the SMG population: galaxies which lie below the detection threshold of the radio observations may have colder dust or lie at $z>3$ (e.g., Ivison et al. 2002; Eales et al. 2003; Swinbank et al. 2008). Accordingly, the conclusions we draw in studying the C05 sample of radio-detected SMGs must properly be restricted to apply only to radio-detected SMGs. However, a wide range of luminosity and redshift is still permitted within the radiodetected sample (see, e.g., Ivison et al. 2002; Blain et al. 2004).

\subsection{IRAC Observations and Data}

We have obtained observations with the IRAC instrument on Spitzer for all seven SMG fields in C05 in all four wavelength channels $(3.6,4.5,5.8$, and $8.0 \mu \mathrm{m})$ from our own General Observer (GO) program with the specific goal of imaging SMGs as well as from publicly available data from various Legacy and Guaranteed Time Observation (GTO) programs obtained through the Spitzer Science Center (SSC) archive. In Table 1, we detail the sources for the IRAC data of each SMG field as well as the central position and sky coverage of the mapped regions we use for our study here. We describe the reduction, mosaicking, and source extraction of these data in Appendix A. The final mosaics for each SMG field have pixels of size 0'.6.

We have measured the flux densities of our SMG sample through 4" diameter apertures using SExtractor version 2.5.0 (Bertin \& Arnouts 1996) and applied wavelength-dependent aperture corrections to obtain the total flux. The measured source fluxes are accurate to better than $10 \%$ for IRAC channels $1(3.6 \mu \mathrm{m})$ and $2(4.5 \mu \mathrm{m})$; in channels $3(5.8 \mu \mathrm{m})$ and 4 $(8.0 \mu \mathrm{m})$, the fluxes are accurate to $\sim 10 \%$ since uncertainties in the aperture corrections tend to be larger at the longer wavelengths. The positions of the detected sources are accurate to $\sim 0.5$ in channels 1 and 2 since the absolute pointing has been aligned to the accuracy of the Two Micron All Sky Survey (2MASS; $\sim 0^{\prime \prime} 15$ ) with an rms of typically 0'.3. In channels 3 and 4 , where absolute pointing refinement was unsuccessful, the source position errors have a more significant contribution from the blind pointing error of IRAC $\left(\sim 0^{\prime \prime} .5\right)$, so we conservatively assume that the positions are accurate to $\sim 1^{\prime \prime}$.

The completeness of the sources extracted from our IRAC mosaics varies by sky field since the depth of coverage varies significantly between fields. The completeness of the extraction from a particular mosaic is difficult to estimate with a single 
Table 1

Data Sources for IRAC Imaging of SMGs

\begin{tabular}{lcccccccc}
\hline \hline Field Name & $\begin{array}{c}\text { Center R.A. }^{\mathrm{a}} \\
(\mathrm{J} 2000.0)\end{array}$ & $\begin{array}{c}\text { Center Decl. }^{\mathrm{a}} \\
(\mathrm{J} 2000.0)\end{array}$ & PID & $\begin{array}{c}\text { Field Size }^{\mathrm{b}} \\
(\operatorname{arcmin} \times \operatorname{arcmin})\end{array}$ & $\begin{array}{c}S_{\text {lim }, 3.6 \mu \mathrm{m}} \mathrm{c} \\
(\mu \mathrm{Jy})\end{array}$ & $\begin{array}{c}S_{\text {lim }, 4.5 \mu \mathrm{m}}{ }^{\mathrm{c}} \\
(\mu \mathrm{Jy})\end{array}$ & $\begin{array}{c}S_{\text {lim }, 5.8 \mu \mathrm{m}}{ }^{\mathrm{c}} \\
(\mu \mathrm{Jy})\end{array}$ & $\begin{array}{c}S_{\text {lim }, 8.0 \mu \mathrm{m}} \\
(\mu \mathrm{Jy})\end{array}$ \\
\hline CFRS-03h & 030244.7 & +000739 & 3473 & $10.0 \times 10.9$ & 0.5 & 1.0 & 6.4 & 9.7 \\
Lockman & 105222.3 & +572454 & $81^{\mathrm{d}}$ & $20.2 \times 22.2$ & 0.4 & 0.7 & 4.8 & 7.2 \\
& & & 1077 & & & & & \\
GOODS-N & 123654.9 & +621419 & $169^{\mathrm{e}}$ & $16.5 \times 10.0$ & 0.1 & 0.1 & 0.8 & 1.1 \\
SSA-13 & 131226.1 & +423910 & $64^{\mathrm{d}}$ & $17.5 \times 14.9$ & 0.9 & 1.8 & 11.9 & 14.5 \\
CFRS-14h & 141745.3 & +522908 & $8^{\mathrm{d}}$ & $8.3 \times 7.1$ & 0.2 & 0.4 & 2.6 & 3.9 \\
ELAIS-N2 & 163647.1 & +410035 & 3473 & $14.2 \times 10.7$ & 0.5 & 0.9 & 6.2 & 7.6 \\
SSA-22 & 221807.9 & +001727 & 3473 & $11.8 \times 8.6$ & 0.4 & 0.8 & 5.9 & 8.1 \\
& 221730.9 & +001340 & $64^{\mathrm{d}}$ & $5.5 \times 5.5$ & 0.2 & 0.5 & 3.4 & 4.5 \\
\hline
\end{tabular}

Notes. Units of R.A. are hours, minutes, and seconds. Units of decl. are degrees, arcminutes, and arcseconds.

${ }^{\text {a }}$ Coordinates of the center of field imaged in all four IRAC channels.

$\mathrm{b}$ The size of field imaged in all four IRAC channels.

${ }^{\mathrm{c}}$ Median theoretical $3 \sigma$ point-source sensitivity in combined mosaic.

${ }^{\mathrm{d}}$ Data from Spitzer Guaranteed Time Observations.

e Data from Spitzer Legacy Project.

number since the depth of imaging also varies within mosaics, especially in the cases in which imaging has been combined from multiple observing programs and PIs. Instead, we give an idea of the typical detection limits for each SMG field in Table 1 by listing the median theoretical $3 \sigma$ point-source sensitivity in each IRAC band calculated using the SSC's sensitivityperformance estimation tool. ${ }^{9}$ For the majority of cases, the median integration time and sensitivity are essentially identical to the mode. The exception is the Lockman East Field, in which the different programs from which we have obtained data obtained imaging to very different depths. In this case, a small $\left(\sim 6^{\prime} \times 6^{\prime}\right)$ portion of our final mosaic is deeper than the rest of the area by approximately a factor of 2 in all the IRAC bands.

\subsection{MIPS Observations and Data}

We have compiled imaging observations for the C05 SMG sample in the $24 \mu \mathrm{m}$ and $70 \mu \mathrm{m}$ bands of MIPS from a variety of observing programs. Data for the CFRS-03h and SSA-22 fields were obtained through our GO program, while data for four of the remaining five SMG fields come from Spitzer Legacy Projects and GTO programs available in the SSC data archive. Unfortunately, the SSA-13 field has no MIPS observations available. ${ }^{10}$ As with the IRAC observations, to make the best use of the data, we use all that is available even though this means that the depth of imaging varies significantly between fields and also within fields, especially in the cases in which imaging from multiple observing programs and PIs has been utilized. In Tables 2 and 3, we detail the data sources, sky coverage, and median $3 \sigma$ point-source sensitivities for the MIPS $24 \mu \mathrm{m}$ and $70 \mu \mathrm{m}$ data, respectively, for each field. We describe the mosaicking and source extraction for the 24 and $70 \mu \mathrm{m}$ data in Appendix B. The final $24 \mu \mathrm{m}$ mosaics have pixels of size 1".275 except in the GOODS-N field where the pixels are 1".2. All of the $70 \mu \mathrm{m}$ mosaics have $4^{\prime \prime}$ pixels.

We measured flux densities for all sources in our mosaics through point-response-function (PRF) fitting using the APEX module within the MOPEX software package (Makovoz \& Marleau 2005). The absolute $24 \mu \mathrm{m}$ fluxes of extracted sources are accurate to within $10 \%$ for bright sources $(\mathrm{S} / \mathrm{N}>20)$;

\footnotetext{
9 http://ssc.spitzer.caltech.edu/tools/senspet/

10 This field was originally in a GTO proposal but was not observed.
}

for fainter sources $(5<\mathrm{S} / \mathrm{N}<10)$ there is an additional systematic $\sim 10 \%-15 \%$ uncertainty in the flux measurements introduced by the extraction procedure (Fadda et al. 2006). We do not include any flux uncertainty due to non-negligible color corrections needed to correct to monochromatic flux densities, since we have chosen not to apply them because redshifted emission and absorption features passing into the $24 \mu \mathrm{m}$ band (e.g., mid-IR PAH features, Menéndez-Delmestre et al. 2007, 2009; Pope et al. 2008) cause the color corrections to vary dramatically with redshift and mid-IR spectrum. At $70 \mu \mathrm{m}$, the measured source fluxes are accurate to $\sim 10 \%$, including color corrections to monochromatic flux density. The errors in the absolute positions of extracted sources in the $24 \mu \mathrm{m}$ images are dominated by the blind pointing accuracy of MIPS and are accurate to within 1".5 (see Appendix B.2); at $70 \mu \mathrm{m}$, the errors in the absolute positions of detected sources are dominated by noise and the positions are accurate to within $4^{\prime \prime}$.

\section{RESULTS OF SPITZER IMAGING OF RADIO-DETECTED SMGS}

\subsection{Identification of SMG Counterparts in Images}

As discussed in Section 1, the identification of submm sources at optical and near-IR wavelengths is problematic due to the large SCUBA beam size $\left(15^{\prime \prime}\right)$ and error circle at $850 \mu \mathrm{m}$ $\left(r \sim 4^{\prime \prime}\right)$ and the faintness of SMGs at optical wavelengths $(R \sim 25)$. Statistical methods based on the probability of finding a source at a given distance from the submm position are often used to determine search radii for SMG counterparts at other wavelengths (e.g., Lilly et al. 1999; Ivison et al. 2002). However, with precise radio positions from $\mathrm{C} 05$, the ambiguity is removed in our mid-IR counterpart selection and statistical methods are unnecessary.

We identify counterparts for each SMG in all four IRAC channels and two MIPS channels separately through their radio positions in $\mathrm{C} 05$. We do not require detection in a particular IRAC band for counterpart identification, as Ashby et al. (2006) do in their $8.0 \mu \mathrm{m}$ selection of SMG counterparts, because this would introduce additional selection effects on our sample. We determine search radii for counterparts by adding in quadrature the typical uncertainty in the radio positions $\left(0{ }^{\prime} 5 ; \mathrm{C} 05\right)$ and the uncertainties in the IRAC and MIPS positions. We thus obtain 1". 1 for our IRAC search radius, $2^{\prime \prime}$ at $24 \mu \mathrm{m}$, and $4^{\prime \prime}$ at $70 \mu \mathrm{m}$. 
Table 2

Data Sources for $24 \mu \mathrm{m}$ MIPS Imaging of SMGs

\begin{tabular}{|c|c|c|c|c|c|c|}
\hline Field Name & $\begin{array}{l}\text { Center R.A. }^{\mathrm{a}} \\
(\mathrm{J} 2000.0)\end{array}$ & $\begin{array}{l}\text { Center Decl. }{ }^{\mathrm{a}} \\
(\mathrm{J} 2000.0)\end{array}$ & PID & $\begin{array}{c}\text { Field Size } \\
(\operatorname{arcmin} \times \text { arcmin })\end{array}$ & $\begin{array}{l}\text { Median } t_{\text {int }}{ }^{\mathrm{c}} \\
(\mathrm{s})\end{array}$ & $\begin{array}{c}3 \sigma \text { Depth } \\
(\mu \mathrm{Jy})\end{array}$ \\
\hline CFRS-03h & 030234.2 & +000909 & 3473 & $12.7 \times 13.3$ & 1308 & 93 \\
\hline Lockman & 105215.0 & +572144 & $\begin{array}{c}81^{\mathrm{e}} \\
1077 \\
142^{\mathrm{f}}\end{array}$ & $22.1 \times 13.6$ & 1771 & 84 \\
\hline GOODS-N & 123654.9 & +621419 & $169^{\mathrm{f}}$ & $18.8 \times 13.4$ & 30458 & 44 \\
\hline CFRS-14h & 141744.0 & +523025 & $8^{\mathrm{e}}$ & $9.7 \times 8.6$ & 681 & 109 \\
\hline ELAIS-N2 & 163647.1 & +405932 & $183^{\mathrm{f}}$ & $17.6 \times 16.5$ & 154 & 143 \\
\hline SSA-22 & 221807.4 & +001733 & 3473 & $10.8 \times 11.5$ & 1227 & 100 \\
\hline & 221730.3 & +001348 & $64^{\mathrm{e}}$ & $7.5 \times 8.2$ & 542 & 131 \\
\hline
\end{tabular}

Notes. Units of R.A. are hours, minutes, and seconds. Units of decl. are degrees, arcminutes, and arcseconds.

${ }^{\text {a }}$ Coordinates of the center of combined MIPS $24 \mu \mathrm{m}$ mosaic.

$\mathrm{b}$ The size of combined mosaic.

${ }^{\mathrm{c}}$ Median integration time per pixel in combined mosaic.

${ }^{\mathrm{d}}$ Median formal $3 \sigma$ point-source sensitivity in combined mosaic.

${ }^{\mathrm{e}}$ Data from Spitzer Guaranteed Time Observations.

${ }^{\mathrm{f}}$ Data from Spitzer Legacy Project.

Table 3

Data Sources for $70 \mu \mathrm{m}$ MIPS Imaging of SMGs

\begin{tabular}{|c|c|c|c|c|c|c|}
\hline Field Name & $\begin{array}{c}\begin{array}{c}\text { Center R.A. } \\
(\mathrm{J} 2000.0)\end{array} \\
\end{array}$ & $\begin{array}{c}\text { Center Decl. }^{\mathrm{a}} \\
(\mathrm{J} 2000.0)\end{array}$ & PID & $\begin{array}{c}\text { Field Size }^{\mathrm{b}} \\
(\operatorname{arcmin} \times \operatorname{arcmin})\end{array}$ & $\begin{array}{c}\text { Median } t_{\text {int }}{ }^{\mathrm{c}} \\
(\mathrm{s})\end{array}$ & $\begin{array}{c}3 \sigma \text { Depth }^{\mathrm{d}} \\
(\mathrm{mJy})\end{array}$ \\
\hline CFRS-03h & 030234.6 & +000850 & 3473 & $13.3 \times 7.8$ & 195 & 10.9 \\
\hline Lockman & 105214.7 & +572138 & $\begin{array}{c}81^{\mathrm{e}} \\
1077 \\
142^{\mathrm{f}}\end{array}$ & $17.4 \times 11.2$ & 970 & 4.6 \\
\hline GOODS-N & 123859.1 & +623436 & $\begin{array}{r}3325 \\
81^{\mathrm{e}}\end{array}$ & $\begin{array}{l}10.0 \times 10.0 \\
85.0 \times 27.2\end{array}$ & $\begin{array}{r}10600 \\
600\end{array}$ & $\begin{array}{l}1.5 \\
6.3\end{array}$ \\
\hline CFRS-14h & 141737.1 & +522806 & $8^{\mathrm{e}}$ & $13.0 \times 7.5$ & 303 & 7.4 \\
\hline ELAIS-N2 & 163648.1 & +405853 & $183^{\mathrm{f}}$ & $14.1 \times 13.8$ & 74 & 8.8 \\
\hline SSA-22 & 221807.8 & $\begin{array}{r}+001720 \\
+001330\end{array}$ & $\begin{array}{r}3473 \\
644^{\mathrm{e}}\end{array}$ & $10.9 \times 6.2$ & $\begin{array}{l}215 \\
653\end{array}$ & $\begin{array}{r}11.8 \\
7.8\end{array}$ \\
\hline
\end{tabular}

Notes. Units of R.A. are hours, minutes, and seconds. Units of decl. are degrees, arcminutes, and arcseconds.

${ }^{\text {a }}$ Coordinates of the center of combined MIPS $70 \mu$ m mosaic.

$\mathrm{b}$ The size of combined mosaic.

${ }^{c}$ Median integration time per pixel for combined mosaic.

${ }^{\mathrm{d}}$ Median formal $3 \sigma$ point-source sensitivity for combined mosaic.

e Data from Spitzer Guaranteed Time Observations.

${ }^{\mathrm{f}}$ Data from Spitzer Legacy Project.

According to the method of Downes et al. (1986), the $24 \mu \mathrm{m}$ search radius corresponds to a $4 \%$ maximum likelihood of a false match in the deepest $24 \mu \mathrm{m}$ mosaic, which also has the highest source density, the GOODS-N mosaic. In several cases, which are noted in Tables 4 and 5, the optical counterpart identified by $\mathrm{C} 05$ is offset from the radio position, and we have allowed a larger offset between the IRAC or MIPS and radio positions for these sources for consistency with $\mathrm{C} 05$.

For $91 \%$ of the SMGs in our sample covered by our IRAC imaging, we find that only one IRAC source lies within the matching radius of 1 ."1, and so source confusion and blending are not problems for counterpart identification in IRAC images (the remaining 9\% are undetected in any IRAC band). At $24 \mu \mathrm{m}$, no more than one source is located within the search radius of any SMG, and $71 \%$ of the $24 \mu \mathrm{m}$ counterparts are located within $1^{\prime \prime}$ of the radio position. However, the $\sim 6^{\prime \prime}$ FWHM of a $24 \mu \mathrm{m}$ point source is sufficiently large that in cases where the submm sources have nearby neighbors, the counterparts can be blended at $24 \mu \mathrm{m}$, and thus it becomes hard to separate the contributions of the different sources to the total $24 \mu \mathrm{m}$ flux.
There are several galaxies in the $\mathrm{C} 05$ sample with near neighbors in high-resolution images (optical, IRAC, or radio), and some of these SMGS are either known to be significantly blended or suspected to be blended with other sources at $24 \mu \mathrm{m}$, which are noted in Table 5. For the blended sources, SMM J105200.22 and SMM J123553.26 estimates of the fluxes of the individual components in the blend made using the IRAC positions of the components as input are available from Ivison et al. (2007) and the $24 \mu \mathrm{m}$ source catalog of R. Chary et al. (2009, in preparation) for the GOODS-N field, respectively. We use these deblended flux estimates here, but caution that the deblended flux estimates for these sources may have larger uncertainties than formally calculated in the source extraction process.

\subsection{Detection Statistics and Flux Measurements}

\subsubsection{IRAC Detection Rates and Measurements of SMGs}

The majority of the SMG sample of $\mathrm{C} 05$ is detected as a point source above a $3 \sigma$ level in all of the IRAC bands. We detect 61 of 67 (91\%) SMGs with imaging coverage at $3.6 \mu \mathrm{m}, 63$ of 
Table 4

IRAC Flux Densities for Chapman et al. (2005) SMG Sample

\begin{tabular}{|c|c|c|c|c|c|c|c|}
\hline Chapman et al. (2005) ID & $z_{\text {spec }}$ & $\begin{array}{c}\text { IRAC R.A. } \\
\text { (J2000.0) }\end{array}$ & $\begin{array}{l}\text { IRAC Decl. } \\
(\mathrm{J} 2000.0)\end{array}$ & $\begin{array}{c}S_{3.6 \mu \mathrm{m}} \\
(\mu \mathrm{Jy})\end{array}$ & $\begin{array}{c}S_{4.5 \mu \mathrm{m}} \\
(\mu \mathrm{Jy})\end{array}$ & $\begin{array}{c}S_{5.8 \mu \mathrm{m}} \\
(\mu \mathrm{Jy})\end{array}$ & $\begin{array}{c}S_{8.0 \mu \mathrm{m}} \\
(\mu \mathrm{Jy})\end{array}$ \\
\hline SMM J030226.17+000624.5 & 0.080 & 030226.17 & +000624.1 & $768 \pm 79$ & $516 \pm 52$ & $465 \pm 47$ & $2280 \pm 230$ \\
\hline SMM J030227.73+000653.5 & 1.408 & 030227.74 & +000653.4 & $75.8 \pm 7.7$ & $81.6 \pm 8.3$ & $61.5 \pm 6.5$ & $63.4 \pm 6.8$ \\
\hline SMM J030236.15+000817.1 & 2.435 & 030236.16 & +000816.8 & $7.9 \pm 0.9$ & $10.0 \pm 1.2$ & $15.3 \pm 2.1$ & $<11.7$ \\
\hline SMM J030238.62+001106.3 & 0.276 & 030238.60 & +001105.4 & $17.1 \pm 2.3$ & $20.3 \pm 2.2$ & $<14.6$ & $<12.6$ \\
\hline SMM J030244.82+000632.3 & 0.176 & 030244.84 & +000632.3 & $9.6 \pm 1.0$ & $7.9 \pm 1.1$ & $<11.5$ & $12.9 \pm 2.2$ \\
\hline SMM J105158.02+571800.2 & 2.694 & 105158.03 & +571800.3 & $54.8 \pm 5.7$ & $57.0 \pm 5.9$ & $45.1 \pm 5.3$ & $54.4 \pm 6.0$ \\
\hline SMM J105200.22+572420.2 & 0.689 & 105200.24 & +572421.2 & $23.1 \pm 2.4$ & $26.6 \pm 2.7$ & $42.0 \pm 4.6$ & $99.2 \pm 10.0$ \\
\hline SMM J105201.25+572445.7 & 2.148 & 105201.19 & +572445.6 & $5.6 \pm 0.8$ & $8.9 \pm 1.0$ & $12.2 \pm 2.2$ & $15.2 \pm 1.9$ \\
\hline SMM J105207.49+571904.0 & 2.689 & 105207.56 & +571904.1 & $10.4 \pm 1.2$ & $13.2 \pm 1.8$ & $<17.0$ & $<16.4$ \\
\hline SMM J105219.15+571858.4 & 2.372 & 105219.10 & +571858.0 & $11.3 \pm 1.4$ & $16.0 \pm 2.3$ & $22.9 \pm 3.1$ & $<12.8$ \\
\hline SMM J105227.58+572512.4 & 2.470 & 105227.55 & +572512.4 & $20.2 \pm 2.2$ & $26.3 \pm 2.7$ & $42.6 \pm 5.0$ & $29.3 \pm 3.1$ \\
\hline
\end{tabular}

Notes. Units of R.A. are hours, minutes, and seconds. Units of decl. are degrees, arcminutes, and arcseconds.

${ }^{a}$ This galaxy was identified by Chapman et al. (2005) as a low- $z$ lens unassociated with the SMG. It has been excluded from the analysis presented here.

$\mathrm{b}$ The redshift of this SMG has been revised in Menéndez-Delmestre et al. (2009).

${ }^{c}$ This SMG lies on the edges of the IRAC images in all fields but is clearly detected.

d This SMG has a near neighbor contaminating the $4^{\prime \prime}$ photometry aperture.

e The redshift of this SMG has been revised in Pope et al. (2008).

f The optical counterpart for this SMG is offset from the radio counterpart, so we have allowed a larger IRAC position offset than the nominal tolerance of 1 ". 1 .

$\mathrm{g}$ This SMG is detected in the $3.6 \mu \mathrm{m}$ channel of IRAC but falls within a column affected by column pull-down and with resulting large uncertainties cause it to be formally undetected.

(This table is available in its entirety in a machine-readable form in the online journal. A portion is shown here for guidance regarding its form and content.)

Table 5

MIPS $24 \mu \mathrm{m}$ and $70 \mu \mathrm{m}$ Results for Chapman et al. (2005) SMG Sample

\begin{tabular}{|c|c|c|c|c|c|c|}
\hline Chapman et al. (2005) ID & $\begin{array}{c}24 \mu \mathrm{m} \text { R.A. } \\
(\mathrm{J} 2000.0)\end{array}$ & $\begin{array}{c}24 \mu \mathrm{m} \text { Decl. } \\
(\mathrm{J} 2000.0)\end{array}$ & $\begin{array}{c}70 \mu \text { m R.A. } \\
(\mathrm{J} 2000.0)\end{array}$ & $\begin{array}{c}70 \mu \mathrm{m} \text { Decl. } \\
(\mathrm{J} 2000.0)\end{array}$ & $\begin{array}{l}S_{24 \mu \mathrm{m}} \\
(\mu \mathrm{Jy})\end{array}$ & $\begin{array}{l}S_{70 \mu \mathrm{m}} \\
(\mathrm{mJy})\end{array}$ \\
\hline SMM J030226.17+000624.5 & 030226.15 & +000623.8 & 030226.02 & +000627.1 & $2140 \pm 210$ & $30.1 \pm 6.7$ \\
\hline SMM J030227.73+000653.5 & 030227.74 & +000653.5 & $\ldots$ & $\ldots$ & $479 \pm 53$ & $<13.6$ \\
\hline SMM J030231.81+001031.3 & $\ldots$ & $\ldots$ & $\ldots$ & $\ldots$ & $<111$ & $<14.8$ \\
\hline SMM J030236.15+000817.1 & $\ldots$ & $\ldots$ & $\ldots$ & $\ldots$ & $<78.2$ & $<10.0$ \\
\hline SMM J030238.62+001106.3 & $\ldots$ & $\ldots$ & $\ldots$ & $\ldots$ & $<108$ & $<12.7$ \\
\hline SMM J030244.82+000632.3 & $\ldots$ & $\ldots$ & $\ldots$ & $\ldots$ & $<83.7$ & $<10.4$ \\
\hline SMM J105151.69+572636.0 & 105151.67 & +572635.7 & $\ldots$ & $\ldots$ & $329 \pm 37$ & $<4.2$ \\
\hline SMM J105155.47+572312.7 & 105155.45 & +572312.8 & $\ldots$ & $\ldots$ & $95.0 \pm 17.8$ & $<3.6$ \\
\hline SMM J105158.02+571800.2 & 105158.08 & +571800.2 & $\ldots$ & $\ldots$ & $232 \pm 29$ & $<4.1$ \\
\hline SMM J105200.22+572420.2 $2^{\mathrm{c}, \mathrm{e}, \mathrm{f}}$ & 105200.24 & +572421.5 & 105200.16 & +572422.7 & $475 \pm 60$ & $6.2 \pm 1.1$ \\
\hline SMM J105201.25+572445.7 & 105201.27 & +572445.9 & $\ldots$ & $\ldots$ & $166 \pm 22$ & $<3.7$ \\
\hline SMM J105207.49+571904.0 & 105207.63 & +571904.4 & $\ldots$ & $\ldots$ & $183 \pm 22$ & $<3.2$ \\
\hline SMM J105219.15+571858.4 & 105219.04 & +571857.9 & $\ldots$ & $\ldots$ & $208 \pm 24$ & $<3.3$ \\
\hline SMM J105227.58+572512.4 & 105227.60 & +572512.8 & $\ldots$ & $\ldots$ & $217 \pm 25$ & $<4.5$ \\
\hline SMM J105227.77+572218.2 & $\ldots$ & $\ldots$ & $\ldots$ & $\ldots$ & $<63.9$ & $<4.1$ \\
\hline
\end{tabular}

Notes. Units of R.A. are hours, minutes, and seconds. Units of declination are degrees, arcminutes, and arcseconds.

${ }^{a}$ This galaxy was identified by Chapman et al. (2005) as a low- $z$ lens unassociated with the SMG. It has been excluded from the analysis presented here.

b This SMG has a mid-IR spectrum from IRS published in Menéndez-Delmestre et al. (2007).

${ }^{c}$ This SMG has a mid-IR spectrum from IRS published in Menéndez-Delmestre et al. (2009).

d This SMG has a mid-IR spectrum from IRS published in Valiante et al. (2007).

e This SMG is detected at $24 \mu \mathrm{m}$ but is blended with another nearby source.

${ }^{\mathrm{f}}$ The $24 \mu \mathrm{m}$ measurement for this SMG has been taken from Ivison et al. (2007).

$\mathrm{g}$ This SMG has a mid-IR spectrum from IRS published in Pope et al. (2008).

${ }^{\mathrm{h}}$ The $24 \mu \mathrm{m}$ measurement for this SMG has been taken from Pope et al. (2006).

i The optical counterpart for this SMG is offset from the radio counterpart so we have allowed a larger offset from the radio position than the nominal $2^{\prime \prime}$ at $24 \mu \mathrm{m}$.

$\mathrm{j}$ This SMG has been allowed a larger offset from the radio position at $24 \mu \mathrm{m}$ due to the difference in resolution of the radio images and the $24 \mu \mathrm{m}$ images.

(This table is available in its entirety in a machine-readable form in the online journal. A portion is shown here for guidance regarding its form and content.)

69 with imaging at $4.5 \mu \mathrm{m}(91 \%), 53$ of 68 with imaging at $5.8 \mu \mathrm{m}(78 \%)$, and 51 of 69 with imaging at $8.0 \mu \mathrm{m}(74 \%)$, as summarized in Table 6. Over the seven SMG fields with IRAC observations $(6,4,5,4)$ SMGs lack IRAC imaging in channels 
$(1,2,3,4)$, respectively. The numbers of galaxies with imaging in the different IRAC bands vary because the field imaged by IRAC channels 1 and 3 is offset by 1.5 from the field observed by IRAC channels 2 and 4. Our IRAC map grids ensure that the central areas of the fields are covered in all four IRAC channels, but if a source falls at the far edge of the mosaic field in channels 1 and 3 (or channels 2 and 4), it may not lie in the mosaic field in channels 2 and 4 (or channels 1 and 3). Overall, four SMGs are not imaged in any IRAC band; 67 SMGs have complete imaging coverage.

We list the positions of the IRAC counterparts and our measured flux density in each IRAC band for all of the SMGs in the sample of C05 in Table 4, including those suspected of being low- $z$ lenses and excluded from the analysis of $\mathrm{C} 05$. The positions listed come from the bluest wavelength of detection, since for the 3.6 and $4.5 \mu \mathrm{m}$ IRAC bands absolute pointing refinement was successful, and we expect the uncertainties in the positions to be smaller. The flux errors listed in each band include a $10 \%$ uncertainty to account for the uncertainty in the absolute calibration, though this is an overestimate of the uncertainty for the measurements in the $3.6 \mu \mathrm{m}$ and $4.5 \mu \mathrm{m}$ channels. Blank entries in Table 4 indicate that the position of that particular galaxy is not covered in our IRAC image mosaics.

\subsubsection{MIPS Detection Rates and Measurements of SMGs}

Of the 59 SMGs in the C05 sample with imaging available at $24 \mu \mathrm{m}, 42(71 \%)$ are detected at $>3 \sigma$ significance, and all are point-like in appearance. Excepting the SSA-13 field, three SMGs in our sample have no $24 \mu \mathrm{m}$ imaging available. The $24 \mu \mathrm{m}$ detection rate across the entire sample is somewhat lower than found in previous studies of SMGs in individual fields. Previous studies of samples of radio-selected SMGs with Spitzer-MIPS at $24 \mu \mathrm{m}$ have resulted in nearly $100 \%$ detection rates: Egami et al. (2004) and Ivison et al. (2004) find that six of the seven SCUBA and MAMBO sources, respectively, with radio detections in the Lockman Hole are detected at $24 \mu \mathrm{m}$, while Frayer et al. (2004b) detect seven of $7>3 \sigma$ SCUBA sources with radio detections in the Spitzer Extragalactic First Look Survey (xFLS). Pope et al. (2006) find that 20 of 21 SMGs with securely identified radio counterparts are detected at $24 \mu \mathrm{m}$, and Ivison et al. (2007) detect 25 of $30(83 \%)$ radio-identified SMGs in their sample. However, two important caveats to the comparison of detection rates are: (1) the different studies all use different criteria for their SMG counterpart identifications; (2) we note from Table 2 that the depth of coverage between our different SMG fields is highly variable, so a lack of detection of a particular SMG in our study does not necessarily indicate that it is intrinsically different from the rest of the sample.

The field with the highest $24 \mu \mathrm{m}$ detection rate in our sample, GOODS-N, has the deepest data, suggesting that deeper $24 \mu \mathrm{m}$ observations in the other SMG fields are likely to detect nearly all SMGs. To examine if the $24 \mu \mathrm{m}$ detection rate in GOODS-N agrees with the other SMG fields when the varying depths are taken into account, we show in Figure 1 the cumulative fraction of objects detected as a function of flux density in each SMG field. The plots indicate that the fraction of SMGs detected at $24 \mu \mathrm{m}$ above a flux level of $\sim 100 \mu \mathrm{Jy}$ (which is the typical depth of the $24 \mu \mathrm{m}$ data in the other fields) in the GOODS-N field is roughly the same as the overall fraction detected in the Lockman and ELAIS-N2 fields. Thus, we conclude that even though the GOODS-N field has some fainter objects in it than are observed in the other SMG fields, the fraction of faint objects is not in
Table 6

Summary of Spitzer Detection Statistics for Radio-detected SMGs from Chapman et al. (2005)

\begin{tabular}{lccc}
\hline \hline Instrument/Wavelength & Number Detected & Number Undetected & No Data \\
\hline IRAC/3.6 $\mu \mathrm{m}$ & 61 & 6 & 6 \\
IRAC $/ 4.5 \mu \mathrm{m}$ & 63 & 6 & 4 \\
IRAC $/ 5.8 \mu \mathrm{m}$ & 53 & 15 & 5 \\
IRAC $/ 8.0 \mu \mathrm{m}$ & 51 & 18 & 4 \\
MIPS $/ 24 \mu \mathrm{m}$ & 42 & 17 & 14 \\
MIPS $/ 70 \mu \mathrm{m}$ & 4 & 55 & 14 \\
\hline
\end{tabular}

excess relative to the other fields and is thus consistent with the GOODS-N field having a higher detection rate due to its deeper data. Overall, we find a median $24 \mu \mathrm{m}$ flux and $1 \sigma$ scatter for our SMG sample of $S_{24}=231 \pm 735 \mu \mathrm{Jy}$, including all sources which are not detected. Our median is comparable to the median for the SMG sample of Pope et al. (2006), $S_{24}=241 \pm 202 \mu \mathrm{Jy}$, suggesting, as expected, that both surveys detect the bulk of the population.

At $70 \mu \mathrm{m}$, a much smaller fraction of the C05 SMG sample is detected than in the IRAC bands or the $24 \mu \mathrm{m}$ band, four out of $59(7 \%)$ with imaging. The four SMGs which are detected at $70 \mu \mathrm{m}, \mathrm{SMM}$ J105200.22 ( $z=0.689)$, SMM J123634.51 $(z=$ 1.219), SMM J141742.04 $(z=0.661)$, and SMM J141741.81 $(z=1.150)$, lie in the low-redshift tail $(z<1.5)$ of the radiodetected SMG redshift distribution, consistent with the results of Huynh et al. (2007). Even in the GOODS-N field, which has the deepest observations of all the SMG fields at $70 \mu \mathrm{m}$, no $z>1.5$ sources are detected, so it is unlikely that the variation in coverage depth between different SMG fields is responsible for the low detection rate. The $70 \mu \mathrm{m}$ detections of the $z<1.5$ sources, however, are good confirmations that the galaxies are the sources of far-IR/submm emission detected by SCUBA, and not misidentifications of higher redshift sources being lensed by a lower redshift galaxy. The detections of these relatively lowredshift SMGs at $70 \mu \mathrm{m}$ and $850 \mu \mathrm{m}$ suggest that these galaxies have cold dust temperatures ( $T_{d} \sim 20-30 \mathrm{~K}$; Paper III).

In Table 5, we present the positions and flux measurements for the counterparts of the C05 SMGs at 24 and $70 \mu \mathrm{m}$. The stated flux errors for each galaxy at each wavelength include a $10 \%$ absolute calibration uncertainty estimate.

\subsection{Stacking Analysis for MIPS Nondetections 3.3.1. $24 \mu \mathrm{m}$-Undetected SMGs}

The SMGs which are not detected at $24 \mu \mathrm{m}$ span a range of redshift from $z \sim 0.2$ to $z \sim 3.5$, having a median $\langle z\rangle=2.4$. To determine an average $24 \mu \mathrm{m}$ flux for the undetected sources and check that our nondetections could be the result of insufficiently deep imaging, we have performed a simple stacking of the $24 \mu \mathrm{m}$ images of the positions of those SMGs. First, we have taken $20^{\prime \prime} \times 20^{\prime \prime}$ cutouts centered on the radio positions of the undetected SMGs from $24 \mu \mathrm{m}$ mosaics in which all sources with $\mathrm{S} / \mathrm{N}>3$ have been subtracted. We then subtracted the median of its pixels from each cutout in the stack to improve the local background removal, then rotated each cutout by $90^{\circ}$ relative to the image before it in the stack. We finally co-added the images in the stack, weighting the images by the rms of their pixels. We statistically detect a $3.7 \sigma$ source in the stacked image with a flux density of $S_{24}=41.5 \pm 11.5 \mu \mathrm{Jy}$, which suggests that deeper imaging will prove fruitful in revealing the $24 \mu \mathrm{m}$ counterparts to the SMGs in our sample which we have not detected. Using the value of the stacked $24 \mu \mathrm{m}$ flux for the 


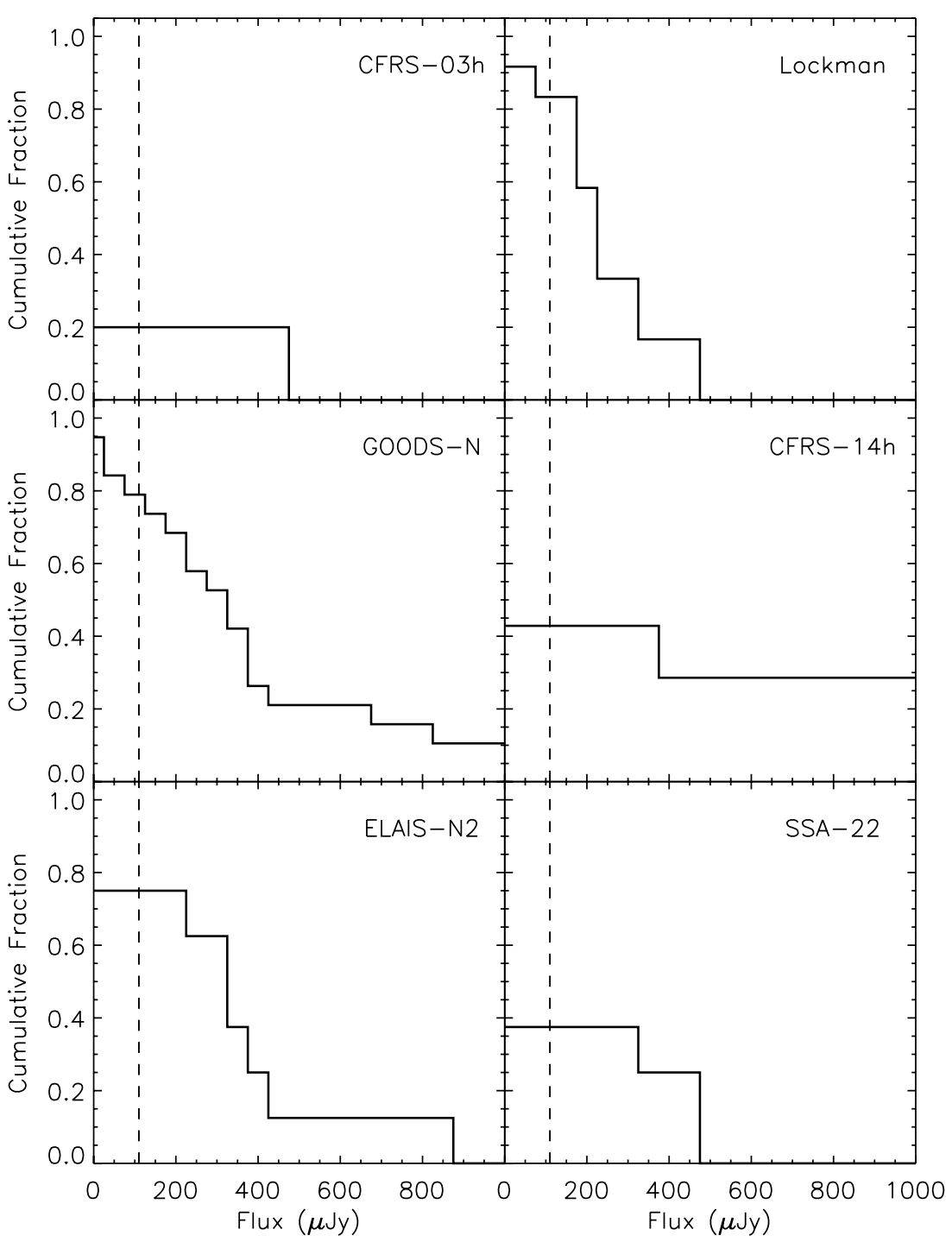

Figure 1. Cumulative fraction of SMGs detected in each field as a function of flux density. The fraction of sources in the GOODS-N field which lies below the detection limits of the other fields is consistent with those not detected in the Lockman Hole field and the ELAIS-N2 field.

undetected galaxies with the median $850 \mu \mathrm{m}$ flux for these same galaxies, $6.1 \mathrm{mJy}$, implies a lower $S_{24} / S_{850}$ ratio and thus redder IR color than the median ratio found for the galaxies which are detected at $24 \mu \mathrm{m}\left(S_{24} / S_{850}=0.007 \pm 0.002\right.$ for undetected SMGs, $\left\langle S_{24} / S_{850}\right\rangle=0.049 \pm 0.040$ for the detected galaxies). For the undetected SMGs at low redshifts, $z<1$, this suggests less dust continuum and a lower dust temperature, while for $z \sim 2$ SMGs the lower ratio suggests less flux in the $7.7 \mu \mathrm{m}$ $\mathrm{PAH}$ feature as well as possibly a cooler dust temperature.

\subsection{2. $70 \mu \mathrm{m}$-Undetected SMGs}

The large number of undetected SMGs provides an important constraint on the IR SEDs of our SMG sample: the limits rule out significant contributions of warm dust $\left(T_{d} \sim 80-100 \mathrm{~K}\right)$ among the $z \sim 2-3$ SMGs in our sample. The upper limits will also be useful for planning future surveys of SMGs with the Photodetector Array Camera and Spectrometer (PACS) on Herschel. Stacking the $70 \mu \mathrm{m}$ images of the undetected SMG positions also provides an idea of an average $70 \mu \mathrm{m}$ flux to expect for these undetected SMGs in future surveys. We have stacked $84^{\prime \prime} \times 84^{\prime \prime}$ cutouts from the $70 \mu \mathrm{m}$ mosaics centered on the radio positions of the undetected SMGs, in which all sources with $\mathrm{S} / \mathrm{N}>3$ have been subtracted. We employ a process similar to that of Huynh et al. (2007), and the same as that used for stacking the $24 \mu \mathrm{m}$ cutouts: we first subtract the median of its pixels from each cutout in the stack to improve the local background removal and then rotate each cutout by $90^{\circ}$ relative to the image before it in the stack before co-adding the images in the stack. The co-addition weights each cutout image by the rms of its pixels. For comparison, we have also constructed a coadded, stacked image of random positions within the mosaics. To represent effectively the different depths of imaging between fields in the random stack, we have used in the random stack the same number of random cutouts from a particular field as the number of undetected SMGs in that field.

In the stacked image of $50 \mathrm{SMG}$ positions, we find a $2.8 \sigma$ detection with a flux density of $S_{70}=0.50 \pm 0.18 \mathrm{mJy}$. For comparison, in the stack of random image positions, no sources with $\mathrm{S} / \mathrm{N}>2$ are found, suggesting that the emission in the stacked SMG image is real. The flux density of the source in our $70 \mu \mathrm{m}$ image stack agrees within the errors with the average flux for SMGs in the stacking analysis of Huynh et al. (2007). However, given the range of redshift of our undetected SMG sources $(0.2<z<3.5)$, it is unclear if this flux density should be interpreted as typical for all of the undetected SMGs or 
just a subset. Huynh et al. (2007) find in their $70 \mu \mathrm{m}$ stacking analysis that the $\sim 3 \sigma$ source found in their stacked image is dominated by galaxies with $z<2$. Accordingly, we divide our stack into separate stacks for SMGs with $z<2$ (20 galaxies) and $z>2$ (30 galaxies) for comparison. Our stacked image of the $z<2$ SMGs contains a marginally detected source $(2.9 \sigma)$ with flux $S_{70}=0.56 \pm 0.19 \mathrm{mJy}$, while the image stack of the $z>2$ SMGs appears dominated by noise (a $12^{\prime \prime}$ aperture placed on the center of the image has a flux of $S_{70}=0.22 \pm 0.18 \mathrm{mJy}$ ). The division of our stack of all SMG positions into $z<2$ and $z>2$ galaxies thus suggests that the full stack is dominated by SMGs with $z<2$.

\section{DISCUSSION}

In this section, we discuss various ways of combining and utilizing the data from IRAC and MIPS to determine if future studies of SMGs can be simplified. In particular, we wish to see if the rest-frame near-IR properties of SMGs show trends with redshift which we may use to identify, at least approximately, the redshift of SMGs. Also, we wish to know if the near-IR colors of SMGs stand out, to permit easier identification of SMG optical and near-IR counterparts and position refinement; due to the limited number of IRAC color combinations we focus on improving tests proposed in the literature based on smaller samples of SMGs. Most importantly, we wish to know if the near- and mid-IR colors of SMGs can betray an obscured AGN which could be powering the tremendous far-IR luminosity. As the near- and mid-IR are wavelength regions where starlight and AGN emission are not hampered by dust, we may be able to use the information provided by IRAC and MIPS to separate stellardominated SMGs from AGN-dominated SMGs. Note that we defer a full UV-near-IR SED analysis to Paper II, however, focusing in this section on trends in IRAC and MIPS-24 $\mu \mathrm{m}$ properties.

\subsection{Mid-IR Colors of SMGs: Trends with Redshift}

\subsubsection{IRAC Colors versus Redshift}

Part of the power of our sample rests in the spectroscopic redshifts available for each galaxy, with which we can identify any color trends with redshift which may help identify the redshifts of SMGs without time-consuming spectroscopy. In Figure 2, we plot rest-frame near-IR colors versus redshift to see if any of the colors can help indicate redshifts of SMGs. We show the binned median color and standard deviation for bins of 11 galaxies in these plots, with the $1 \sigma$ scatter shown as the error bar, to assist in identifying trends. However, no significant trends of mid-IR color with redshift are visible for the SMGs. In fact, the spread in the IRAC colors of the radio-detected SMGs, especially those at $z>2$, is relatively tight, less than an order of magnitude; the spread in the $S_{4.5} / S_{3.6}$ color is especially small. We find the medians and standard deviations of the different near-IR colors to be $\left\langle S_{3.6} / S_{K}\right\rangle=2.38 \pm 1.28,\left\langle S_{4.5} / S_{3.6}\right\rangle=$ $1.27 \pm 0.24,\left\langle S_{5.8} / S_{3.6}\right\rangle=1.77 \pm 0.74,\left\langle S_{5.8} / S_{4.5}\right\rangle=1.31 \pm 0.46$, $\left\langle S_{8.0} / S_{4.5}\right\rangle=1.37 \pm 0.89$, and $\left\langle S_{8.0} / S_{5.8}\right\rangle=1.14 \pm 0.56$. The sources included in these statistics have IRAC detections in at least one of the filters in the flux ratio: we have excluded in the medians the sources which have upper limits in both filters of the flux ratio since the ratios are unconstrained.

Such small scatter in the IRAC colors is in marked contrast with the observed $I-K$ and $J-K$ colors for this same sample of SMGs found by Smail et al. (2004), which span 4-5 mag, and with the results of Frayer et al. (2004a). That

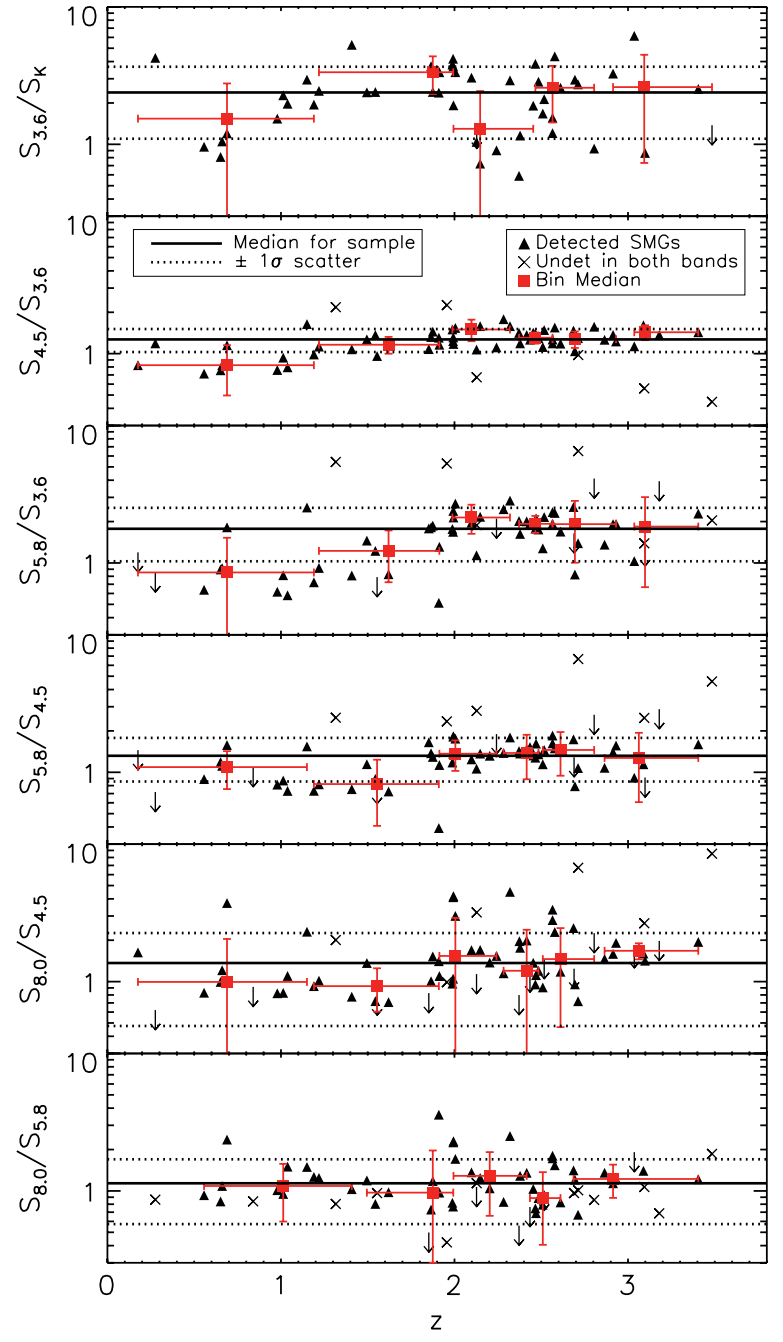

Figure 2. IRAC colors vs. redshift for the C05 sample of radio-detected SMGs. Downward-pointing arrows represent objects which are detected in only one band. We also plot the binned median flux for independent 11 object bins, centered at the indicated redshifts. The median colors of the entire sample and the $\pm 1 \sigma$ ranges are marked. We conclude that SMGs are more uniform in IRAC colors than in optical/near-IR band colors.

(A color version of this figure is available in the online journal.)

SMGs appear more uniform in their rest-frame near-IR colors than in their rest-frame optical colors likely reflects the waning influence of dust obscuration in the near-IR as compared with the effects of extinction at optical wavelengths. While it is possible that some selection bias toward bluer galaxies exists in our sample due to the presence of a spectroscopic redshift from optical wavelengths, given the similarity of the rest-frame nearIR colors across the range of optical extinction, it is unlikely that a selection bias will affect the trends in the mid-IR colors.

\subsubsection{Identifying $z>1.5$ SMGs Through a Mid-IR Color-Magnitude Diagram}

Pope et al. (2006) suggest that a $5.8 \mu \mathrm{m}$ versus $5.8 / 3.6 \mu \mathrm{m}$ ratio color-magnitude diagram can be used to separate $z>1.5$ SMGs from lower- $z$ SMGs, even if a source contains an X-raydetected AGN. However, they relied on photometric redshifts for their analysis, and any spectroscopic redshifts came from our current sample from C05. With a larger sample of SMGs with complete spectroscopic redshifts, we can unambiguously evaluate the ability of the $5.8 \mu \mathrm{m}-5.8 / 3.6 \mu \mathrm{m}$ color-magnitude 


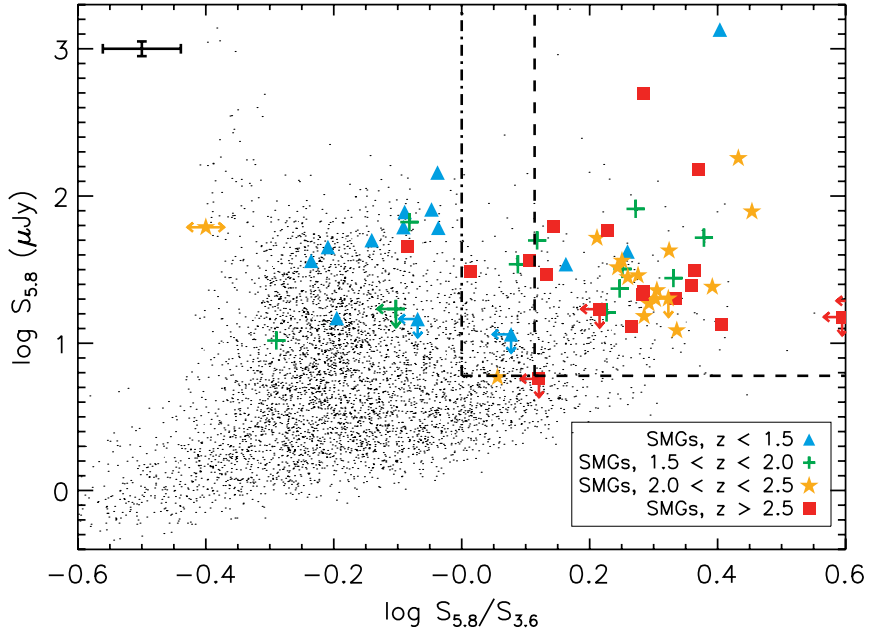

Figure 3. $5.8 \mu \mathrm{m}$ vs. $5.8 \mu \mathrm{m} / 3.6 \mu \mathrm{m}$ color-magnitude diagram for radiodetected SMGs in our sample and field galaxies from the GOODS-N sample. Field galaxies are represented by black dots. SMGs are are divided into bins according to redshift. Dashed lines indicate the region of the plot identified by Pope et al. (2006) as containing SMGs at $z>1.5$; dot-dashed lines represent the relaxed criteria we propose. A typical error bar for the SMGs is shown in the upper left corner. Six SMGs are missing from this plot because we cannot obtain useful constraints on their $S_{5.8} / S_{3.6}$ flux ratios due to upper limits. Two additional SMGs are absent from the plot because the galaxies fall outside the field of view of the images. One SMG is missing a $3.6 \mu \mathrm{m}$ flux due to local noise in the image and has been plotted at the arbitrary color $\log \left(S_{5.8} / S_{3.6}\right)=-0.4$ The color-magnitude selection criteria suggested by Pope et al. (2006) correctly categorize $83 \%$ of the SMG sample as $z<1.5$ or $z>1.5$ galaxies.

(A color version of this figure is available in the online journal.)

diagram to select $z>1.5$ SMGs. In Figure 3, we plot the appropriate diagram for field galaxies (i.e., all sources which are not SMGs) in the deep images of the GOODS-N field and radio-detected SMGs from the C05 sample with detections at $3.6 \mu \mathrm{m}$, color- and shape-coding the SMGs by redshift bin. We indicate the region in which Pope et al. (2006) find that 11/ 11 SMGs in their sample have $z>1.5, \log S_{5.8}>0.75$ and $\log \left(S_{5.8} / S_{3.6}\right)>0.1$.

The demarcated region in color-magnitude space does tend to contain SMGs with $z>1.5$; however, there are exceptions. Nine of the $51 z>1.5$ SMGs (17\%) have detections or upper limits which are bluer and fainter than the high- $z$ selection criteria, while three $z<1.5$ SMGs $(23 \%$ of $z<1.5$ SMGs) meet the requirements for high- $z$ designation. Two of the lower- $z$ SMGs falling in high- $z$ region of the plot, SMM J105200.22 $(z=0.689)$ and SMM J141741.81 $(z=1.150)$, have been identified as possible AGN-dominated sources based on their IRAC SEDs by Egami et al. (2004) and Ashby et al. (2006), respectively, and the contribution of an AGN to the near-IR emission could certainly redden the 5.8/3.6 $\mu \mathrm{m}$ color in these galaxies. The other $z<1.5$ SMG located in the high- $z$ region in Figure 3, SMM J163639.01 at $z=1.488$, lies at the $z \sim 1.5$ dividing line, and we choose to consider this particular SMG as a successfully identified $z=1.5$ galaxy rather than a $z<1.5$ galaxy contaminating the high- $z$ identifications. Thus, overall the 5.8/3.6 $\mu \mathrm{m}-5.8 \mu \mathrm{m}$ color-magnitude diagram suggested by Pope et al. (2006) successfully identifies 43 of 52 radio-detected SMGs $(83 \%)$ as having $z>1.5$, with a $z<1.5$ contamination rate of the color-magnitude criteria of $4 \%$ (two out of the 45 SMGs meeting the $z>1.5$ color-magnitude criteria are $z<1.5$ galaxies)

If we relax the 5.8/3.6 $\mu \mathrm{m}$ color criterion for the high- $z$ box to $S_{5.8} / S_{3.6}>1.0$, as shown in Figure 3, allowing for larger- than-typical photometric errors (note typical error bar for the SMGs in the upper left of Figure 3) and SEDs of $z \sim 1.5$ SMGs which decline steeply redward of a rest-frame $1.6 \mu \mathrm{m}$ peak, four additional $z>1.5$ SMGs will be included in the $z>1.5$ region. Then, the success rate of the color-magnitude method of low-/high-redshift classification will improve to $90 \%$, while the $z<1.5$ contamination rate will rise only modestly to $6 \%$.

We suggest that our modification of the 5.8/3.6 $\mu \mathrm{m}-5.8 \mu \mathrm{m}$ color-magnitude criteria proposed by Pope et al. (2006) will be useful in future SMG surveys for separating out high- $z$ SMGs from $z<1$ SMGs for follow-up studies, given its high accuracy and low contamination by low- $z$ galaxies. The criteria may fail when $z<1.5$ sources have a powerful obscured AGN, but it may be possible to differentiate $z<1.5$ AGN from high- $z$ galaxies by examination of the SED over a broader range of wavelength (e.g., optical-mid-IR wavelengths).

\subsection{Separating SMG Counterparts from Faint Field Galaxies in Deep Surveys}

With smaller samples of SMGs, Ashby et al. (2006), Pope et al. (2006), and Yun et al. (2008) found that SMGs tend to be redder than field galaxies in colors formed from various IRAC bands, and suggest that this trait can be used to find SMG counterparts. Here, we extend our near-IR color analysis to a sample of SMGs double the size of any of these previous studies and evaluate several different methods of using IRAC colors to pick out SMG counterparts from field galaxies which may be used in future surveys. Moreover, we have spectroscopic redshifts for all and so can look for any trends in identification with redshift.

Our analysis in this section uses the radio identifications of our SMG sample as a check of the accuracy of different methods of SMG counterpart selection. Note that our comparison to the radio counterpart precludes a proper comparison of the IRAC counterpart selection methods to the method of radio counterpart identification. In our evaluations, we examine the IRAC sources within an $8^{\prime \prime}$ radius of our SMGs, a typical search radius for SMG counterparts, apply the selection criteria proposed by Pope et al. (2006) and Yun et al. (2008), and compare the galaxy suggested by the criteria to the known radio counterpart. We regard a counterpart identification as successful if a single galaxy is selected by the IRAC criteria (i.e., the galaxy meets the colormagnitude or color-color criteria) which agrees with the radioidentified SMG counterpart listed in Table 4. An identification is considered failed if no galaxy is selected within the search radius (unless the known radio counterpart was undetected in our IRAC images) or if the galaxy selected by the IRAC criteria is not the same as the radio counterpart. An "ambiguous" identification is one where multiple galaxies within the search radius, including the known radio counterpart, meet the IRAC color criteria and more information would be required to determine the correct counterpart. Note that we include galaxies in our statistics which are undetected and have upper limits in one or more IRAC bands, since the upper limits can be consistent with the selection criteria. For ease of reference, we summarize the results of both counterpart identification methods in Table 7.

\subsubsection{SMG Counterpart Selection Through a 5.8/3.6 $\mu \mathrm{m}-5.8 \mu \mathrm{m}$ Color-Magnitude Diagram}

We look once more at the 5.8/3.6 $\mu \mathrm{m}-5.8 \mu \mathrm{m}$ colormagnitude diagram in Figure 3. Pope et al. (2006) suggest that the same color-magnitude criteria used to separate $z>1.5$ SMGs from lower- $z$ SMGs can be used as a simple cut to select 
Table 7

Summary of Results of IRAC SMG Identification Methods Within $8^{\prime \prime}$ Search Radius

\begin{tabular}{lccc}
\hline \multicolumn{1}{c}{ Method of Counterpart ID } & $\begin{array}{c}\text { Unique, Correct } \\
(\%)\end{array}$ & $\begin{array}{c}\text { Ambiguous } \\
(\%)\end{array}$ & $\begin{array}{c}\text { None }^{\mathrm{b}} \text { or Incorrect }^{\mathrm{c}} \\
(\%)\end{array}$ \\
\hline Pope et al. (2006) & 53 & 18 & 29 \\
Pope et al. (2006), $z>1.5$ only & 63 & 19 & 19 \\
Pope et al. (2006), Relaxed & 56 & 21 & 23 \\
Pope et al. (2006), Relaxed and $z>1.5$ only & 68 & 21 & 11 \\
Yun et al. (2008) & 62 & 35 & 3 \\
\hline
\end{tabular}

Notes. Fractions listed include SMGs undetected by IRAC for which the upper limits are available.

a A "correct" counterpart refers to one which agrees with the radio counterpart.

b Multiple galaxies within the search radius meet the IRAC counterpart selection criteria.

c An "incorrect" counterpart is one which meets the IRAC selection criteria, but disagrees with the radio counterpart.

mid-IR counterparts to SMGs since a galaxy with colors meeting those criteria has a low probability of occurring at random within an $8^{\prime \prime}$ search radius. A quick visual comparison of our IRAC data for a large SMG sample to field galaxies from the deep GOODS$\mathrm{N}$ data set in Figure 3 provides encouragement that the colormagnitude criteria $\log \left(S_{5.8} / S_{3.6}\right)>0.1$ and $\log S_{5.8}>0.75$ will function as SMG selection criteria: almost $70 \%$ of the SMGs in our sample are found within this region of color-magnitude space, whereas less than $6 \%$ of non-SMG field galaxies in GOODS-N lie in this region. However, it is important to recall from Section 4.1.2 that SMGs which fall within the desired region of color-magnitude space are preferentially located at $z>1.5$; so, in using the color-magnitude criteria of Pope et al. (2006) we will be biased against the identification of counterparts for SMGs at $z<1.5$. Below, we attempt to illustrate the impact of the identification bias by compiling success/failure statistics for both our full sample of radiodetected SMGs and the $z>1.5$ subsample.

When we apply the $5.8 \mu \mathrm{m}-5.8 / 3.6 \mu \mathrm{m}$ flux and color cuts of Pope et al. (2006) to the IRAC sources within an $8^{\prime \prime}$ radius of the radio position of each SMG in our sample, we find that the IRAC color-magnitude selection successfully identifies $53 \%$ of the radio-selected SMGs with the correct, unique counterpart. The IRAC selection criteria fail to correctly identify the radio counterpart (or any counterpart) for $29 \%$ of our sample. If we consider the only the $z>1.5 \mathrm{SMGs}$, so that the selection bias against $z<1.5$ galaxies cannot affect the success statistics, $63 \%$ of the SMGs are successfully connected to the correct, unique counterpart, while the fraction of failed identifications drops to $19 \%$. For a separate $18 \%$ of our sample (19\% if only the $z>1.5 \mathrm{SMGs}$ are considered), using the IRAC criteria results in ambiguous identifications; for comparison, the fraction of our SMG sample with ambiguous radio counterparts (i.e., double radio source identifications in $\mathrm{C} 05$ ) is $12 \%$. We note that the subsample of our SMGs having ambiguous IRAC counterparts does not completely overlap with the subsample with ambiguous radio counterparts: while the SMGs for which the double radio sources have the same redshift all have ambiguous IRAC identifications, the radio-ambiguous SMGs for which one of the radio sources lies at $z<0.5$ do not have ambiguous IRAC identifications.

If we relax the 5.8/3.6 $\mu \mathrm{m}$ color criterion to $S_{5.8} / S_{3.6}>1.0$, as suggested in the previous section to increase the success of the redshift classifications, the fraction of our SMG sample for which the modified IRAC selection criteria successfully identify the unique counterpart increases to $56 \%$. This fraction increases again to $68 \%$ if we consider only the $z>1.5$ subsample of our SMGs. The fraction of failed identifications under the modified color-magnitude criteria decreases to $23 \%$, which falls to $11 \%$ when only the $z>1.5 \mathrm{SMGs}$ are considered. The fraction of our sample with ambiguous IRAC-selected counterparts increases modestly to $21 \%$ using the modified criteria, for both the full SMG sample and the $z>1.5$ subsample.

It is thus clear that a candidate mid-IR SMG counterpart falling in the region $\log S_{5.8}>0.75$ and $\log \left(S_{5.8} / S_{3.6}\right)>0.1$ in Figure 3 is more likely to be the correct radio counterpart than one which does not. However, radio-detected SMGs with $z<1.5$ will be underrepresented in samples selected through these IRAC color-magnitude criteria, which suggests that this method of counterpart selection will be useful in a crude manner to select galaxies for follow-up observations (especially since the ambiguous identifications can likely be resolved through the follow-up observations), but not to confidently identify counterparts for fully representative samples of radio-SMGs.

\subsubsection{SMG Selection Through a $S_{8.0 \mu \mathrm{m}} / S_{4.5 \mu \mathrm{m}}-S_{5.8 \mu \mathrm{m}} / S_{3.6 \mu \mathrm{m}}$ Color-Color Diagram}

Yun et al. (2008) propose to use the $S_{8.0 \mu \mathrm{m}} / S_{4.5 \mu \mathrm{m}}-$ $S_{5.8 \mu \mathrm{m}} / S_{3.6 \mu \mathrm{m}}$ color-color diagram, also used by Lacy et al. $(2004,2007)$ to identify obscured AGN, to separate SMG counterparts from field galaxies. In Figure 4 , we plot the $S_{8.0} / S_{4.5}$ $S_{5.8} / S_{3.6}$ color-color diagram for faint field galaxies in the GOODS-N field (i.e., those sources which are not known to be SMGs) and overplot our SMG sample. We indicate the region in color space that Yun et al. (2008) suggest using for SMG counterpart identification, $\log \left(S_{8.0} / S_{4.5}\right)>-0.3$, $\log \left(S_{5.8} / S_{3.6}\right)>-0.3$, and $\log \left(S_{8.0} / S_{4.5}\right)<\log \left(S_{5.8} / S_{3.6}\right)-0.4$. All but one of the SMGs in our sample fall within the demarcated region of color space. Yet, the odds of a source falling in this region at random are also high: $63 \%$ of the field galaxies in GOODS-N also fall within the portion of color space which SMG counterparts are found in. Such a high fraction of field sources suggests that the criteria proposed by Yun et al. (2008) may be too general to be useful in finding unique SMG counterparts in IRAC data, given the relatively low surface density of SMGs compared to the general field.

When we look in detail at the galaxies in our IRAC images within an $8^{\prime \prime}$ radius of our radio-detected SMGs which meet the color criteria of Yun et al. (2008) and are thus picked out as possible SMG counterparts, the effects of having a large overlap with general field galaxies are clear. The radio-selected SMG counterpart is correctly selected as the unique counterpart by the IRAC color criteria for $62 \%$ of our sample, and for only $3 \%$ of our SMGs does the color selection fail to identify the radio counterpart as the IRAC counterpart. The fraction of the radio-detected SMGs with ambiguous identifications, 


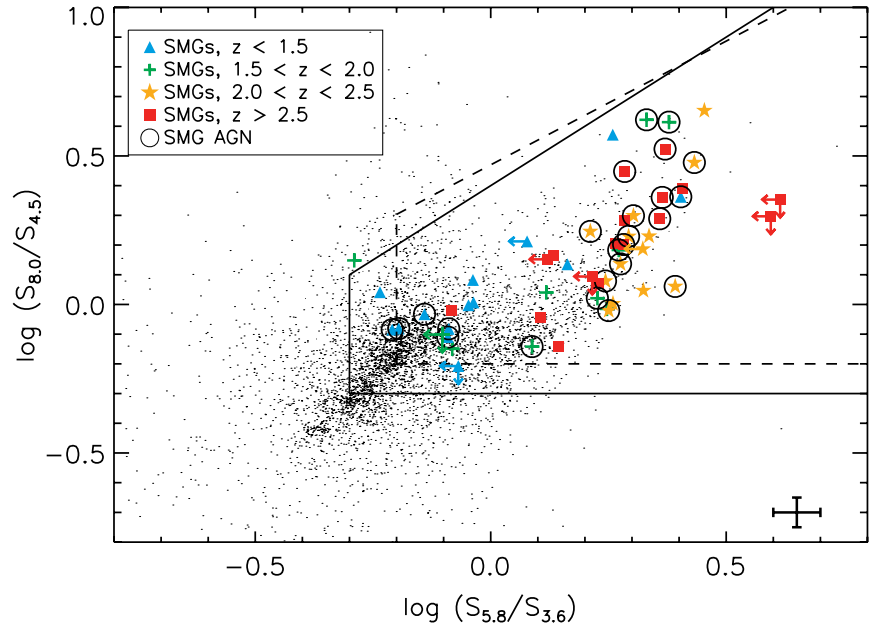

Figure 4. $8.0 \mu \mathrm{m} / 4.5 \mu \mathrm{m}$ vs. $5.8 \mu \mathrm{m} / 3.6 \mu \mathrm{m}$ color-color diagram for SMGs in our sample and field galaxies from GOODS-N. Symbols are as in Figure 3. The dashed lines represent the color-selection criteria of Lacy et al. (2004), while the solid lines indicate the color selection criteria of Yun et al. (2008). SMGs known to contain an AGN from data at any wavelength are circled. A typical error bar for the SMGs is shown in the lower right corner. Six SMGs are missing from this plot because we cannot obtain useful constraints on their $S_{5.8} / S_{3.6}$ flux ratio due to upper limits at the necessary wavelengths, and four more SMGs are absent from the plot because we were unable to measure the flux in one or more of the required bands due to local noise in the images or the galaxy falling out of the field of view of the image. Nearly all of the SMGs in the plot meet the color criteria for SMG counterpart identification proposed by Yun et al. (2008); however, a large fraction of field galaxies also meet the selection criteria, causing $35 \%$ of our sample to have a neighbor within an $8^{\prime \prime}$ radius which also meets the color criteria.

(A color version of this figure is available in the online journal.)

however, is $35 \%$, reflecting the high number of field galaxies which also meet the color selection criteria. The lack of a unique counterpart for such a large fraction of our sample implies that considerable effort and time will be required after the IRAC color-color selection of counterparts to resolve the ambiguous identifications. Hence, even though the rates of success and failure for identifying the entire SMG sample using the $S_{8.0} / S_{4.5}-S_{5.8} / S_{3.6}$ color criteria of Yun et al. (2008) seem better than we found for the color-magnitude criteria of Pope et al. (2006) in Section 4.2.1 above, we suggest that the color criteria of Yun et al. (2008) will not exclude enough field galaxies to be efficient at identifying mid-IR counterparts for large numbers of radio-detected SMGs.

\subsection{Using Spitzer Data to Separate Starburst-dominated and AGN-dominated SMGs}

An important unresolved issue in determining the contribution of SMGs to the star formation history of the universe is the contribution to the far-IR emission of SMGs from active nuclei. As the fraction of SMGs which contain AGNs is now known to be high (Alexander et al. 2005b; Menéndez-Delmestre et al. 2009), the critical step in studying the relative contributions of star formation and AGN activity to the total IR luminosity now is to identify which SMGs contain AGNs which dominate their bolometric output. This is not a trivial step, even with spectroscopic data, as the studies of $\mathrm{C} 05$, Swinbank et al. (2004), and Takata et al. (2006) have shown, or even with Xray observations, as shown by Alexander et al. (2005a). With the advent of Spitzer, numerous authors (e.g., Lacy et al. 2004; Ivison et al. 2004; Stern et al. 2005; Weedman et al. 2006) have proposed methods of separating powerful AGNs from strongly star-forming galaxies in the general galaxy population based on mid-IR colors and fluxes. Several authors (e.g., Egami et al. 2004; Ivison et al. 2004, 2007; Ashby et al. 2006) have suggested that such mid-IR techniques can be used to identify powerful AGN in SMGs as well. In the following sections, we apply several of the diagnostics suggested in the literature to our sample of SMGs to determine which of our sample of radio-detected SMGs contain energetically dominant AGNs, and compare the results to the spectral classifications available from the optical, near-IR, and mid-IR spectra, and from X-ray detections.

\subsubsection{Using IRAC Colors to Identify Obscured AGN-Dominated SMGs}

Lacy et al. $(2004,2007)$ use a $8.0 \mu \mathrm{m} / 4.5 \mu \mathrm{m}-5.8 \mu \mathrm{m} /$ $3.6 \mu \mathrm{m}$ diagram, the same diagram discussed in Section 4.2.2, to discriminate obscured AGNs from bluer field galaxies. In the $S_{8.0} / S_{4.5}-S_{5.8} / S_{3.6}$ color space, field galaxies fall into a clump in the range $[-0.5,0]$ in both axes. Two plumes extend redward in $S_{8.0} / S_{4.5}$, one of which is bluer in $S_{5.8} / S_{3.6}$ and one which is red. Lacy et al. $(2004,2007)$ identify the red plume with obscured AGNs and demarcate a region in color-color space which they use to identify powerful AGNs. In this section, we return to Figure 4 to discuss its use in identifying powerful obscured AGNs in SMGs. In the figure, we indicate the region in color space that Lacy et al. $(2004,2007)$ associate with obscured AGNs; the selected region of color space is slightly different from that proposed for SMG identification by Yun et al. (2008). We have also highlighted in the diagram all of the SMGs which have been classified as AGNs at any wavelength.

The majority of our sample of radio-detected SMGs fall into the region of color space Lacy et al. $(2004,2007)$ identify with powerful obscured AGNs, similar to the findings of Yun et al. (2008). While we might expect such a color-color diagram to reveal AGNs in some SMGs which have not previously been shown to contain one (especially considering that not all of the SMGs have similar quantities of observational data), the large fraction of our sample falling within the demarcated AGN region disagrees strongly with prior estimates of the fraction of the SMG population hosting an energetically dominant AGN (at most 28\%-50\%; Alexander et al. 2003, 2005b). Of course, the disagreement between different indicators does not exclude the possibility that most of the SMGs in our sample contain obscured AGNs.

Sajina et al. (2007a) argued that the $S_{8.0} / S_{4.5}-S_{5.8} / S_{3.6}$ criteria are unreliable at $z>2$ because light from the host galaxies can dominate the AGN near the location of the stellar bump at $1.6 \mu \mathrm{m}$, suggesting that it may be premature to conclude that most of the SMGs in our sample are dominated by powerful obscured AGNs based on continuum colors. Recently, Yun et al. (2008) found that colors in the obscured AGN region of $S_{8.0} / S_{4.5}-S_{5.8} / S_{3.6}$ color space can be produced by high-redshift starburst galaxies in addition to obscured AGN, reinforcing the conclusion of Sajina et al. (2007a) that the $S_{8.0} / S_{4.5}-S_{5.8} / S_{3.6}$ criteria of Lacy et al. $(2004,2007)$ are unreliable for high- $z$ galaxies. Since our sample of SMGs is composed of high- $z$ galaxies which are likely to be starbursts, we hesitate to draw the conclusion that any of these galaxies are dominated by powerful AGN simply because they fall into the AGN region of the $S_{8.0} / S_{4.5}-S_{5.8} / S_{3.6}$ color-color plot.

\subsubsection{Using $24 \mu m-I R A C$ Colors to Identify AGN-dominated SMGs}

Ivison et al. (2004) proposed using a diagram of the ratio $S_{24 \mu \mathrm{m}} / S_{8 \mu \mathrm{m}}$ plotted versus the ratio $S_{8 \mu \mathrm{m}} / S_{4.5 \mu \mathrm{m}}$ to distinguish 


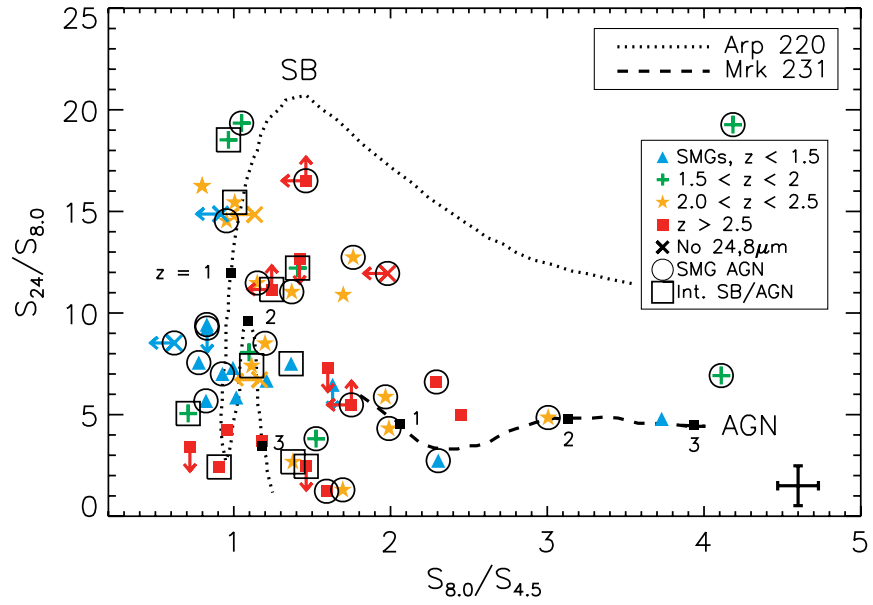

Figure 5. $S_{24 \mu \mathrm{m}} / S_{8 \mu \mathrm{m}}$ vs. $S_{8 \mu \mathrm{m}} / S_{4.5} \mu \mathrm{m}$ color-color plot proposed by Ivison et al. (2004) to separate powerful AGNs from strongly star-forming systems for SMGs. Symbols are as in Figure 3. Diagonal crosses represent SMGs with upper limits at all three wavelengths. The dotted line represents the color-color track of Arp 220 as its SED is redshifted, while the dashed line represents the redshifted color track for Mrk 231. Points along these tracks corresponding to the observed colors when the SEDs are redshifted to $z=(1,2,3)$ are marked. A typical error bar for the SMGs is shown in the lower right corner. Of the six SMGs which are identified as AGN-dominated in the plot and have IRS spectra, five show strong AGN-like mid-IR continuum.

(A color version of this figure is available in the online journal.)

star-forming galaxies from AGN-dominated sources and possibly even distinguish redshift. The idea is based on the observation that the SEDs of two relatively nearby ULIRGs, Arp 220 and Mrk 231, which are frequently used as templates for "Starburst ULIRGs" and "AGN-dominated ULIRGs," cause the two galaxies to follow well-separated tracks in $S_{24 \mu \mathrm{m}} / S_{8 \mu \mathrm{m}}-S_{8 \mu \mathrm{m}} / S_{4.5 \mu \mathrm{m}}$ color space as the SEDs are redshifted. Pope et al. (2008) find that in this particular diagram, the division between starburstand AGN-dominated sources generally agrees with their mid-IR spectral classifications. Since we now have both MIPS-24 $\mu \mathrm{m}$ and IRAC data for most of the radio-detected SMGs in the C05 sample, many of which have separate UV and optical spectral classifications, we can test the effectiveness of Ivison et al. (2004)'s color-color diagram in exposing AGNs. Since we have secure spectroscopic redshifts for our sample, we can also test the use of the color-color diagram as a redshift indicator.

We plot the color-color diagram of Ivison et al. (2004) in Figure 5, including only the SMGs with $24 \mu \mathrm{m}$ observations (we lack information for the galaxies in the SSA 13 field). In the diagram, we have highlighted those galaxies known to show AGN features in their rest-frame UV, optical, or IR spectra, or from X-ray observations, as well as galaxies with intermediate starburst/AGN spectral signatures. We have also plotted the color-color tracks for Arp 220 and Mrk 231 for redshifts corresponding to $z=0-3$ from Ivison et al. (2007). The inflections in the track for Arp 220 are caused by mid-IR spectral features.

In the figure, we find that our SMG sample shows the same general distribution across the color-color space as the sample of SMGs from Ivison et al. (2007). At a glance, however, it is apparent that the diagram will not be a good way to distinguish the redshift of SMGs: galaxies with similar redshifts are scattered over the plot and do not appear to make a clear sequence as the tracks of Arp 220 and Mrk 231 would suggest. This finding supports the suggestion of Sajina et al. (2005) that because of large variations in $24 \mu \mathrm{m}$ flux resulting from strong
Table 8

SMGs Identified as AGN-Dominated Galaxies in Color Diagrams

\begin{tabular}{lc}
\hline \hline Ivison et al. (2004) Diagram & $J-K$ vs. $H-K$ Diagram \\
\hline SMM J105155.47+572312.7 & SMM J030238.62+001106.3 \\
SMM J105200.22+572420.2 & SMM J123600.15+621047.2 \\
SMM J123600.15+621047.2 & SMM J123632.61+620800.1 \\
SMM J123606.72+621550.7 & SMM J123635.59+621424.1 \\
SMM J123616.15+621513.7 & SMM J131215.27+423900.9 \\
SMM J123632.61+620800.1 & SMM J131222.35+423814.1 \\
SMM J123635.59+621424.1 & SMM J131232.31+423949.5 \\
SMM J141741.81+522823.0 & SMM J141741.81+522823.0 \\
$\ldots$ & SMM J163706.51+405313.8
\end{tabular}

Note. ${ }^{\text {a }}$ No previous spectral AGN identification.

mid-IR spectral features high values of the ratio $S_{24 \mu \mathrm{m}} / S_{8 \mu \mathrm{m}}$ do not necessarily indicate low redshifts.

However, the diagram seems useful to identify AGNdominated galaxies: six SMGs with previously identified AGNs fall on the AGN track, along with two SMGs not previously identified as AGN,${ }^{11}$ which we summarize in Table 8 . Five of the six SMGs identified as powerful AGNs in the plot which have midIR spectra from Spitzer (SMM J105155.47, SMM J123600.15, SMM J123616.15, SMM J141741.81, and SMM J163706.51) display AGN continuum in their spectra; four of these six are continuum-dominated. Mid-IR spectra for the two SMGs without IRS spectra predicted to be AGN-dominated will help us confirm the predictive power of the $S_{24} / S_{8.0}-S_{8.0} / S_{4.5}$ diagram.

The SMG in our sample which the $S_{24} / S_{8.0}-S_{8.0} / S_{4.5}$ diagram classifies as a powerful AGN but shows no obvious AGN in its mid-IR or optical spectrum is SMM J105200.22. Curiously, both Egami et al. (2004) and Ivison et al. (2004) suggest also that it should be called an AGN on the basis of its mid-IR colors; yet its mid-IR spectrum from IRS appears to be starburstdominated, with strong PAH features and a mid-IR spectral index of $\alpha_{\text {MIR }}=1.76$ (Menéndez-Delmestre et al. 2009). However, SMM J105200.22 is one of the few SMGs in our sample which is clearly blended with another galaxy at $24 \mu \mathrm{m}$; thus, the difference between the photometric and spectroscopic classifications may reflect incorrect deblending in the $24 \mu \mathrm{m}$ photometric measurement, or multiple sources falling on the IRS slit. Higher spatial resolution mid-IR spectroscopy will be required to resolve the conflict.

Note that not all of the SMGs with identified AGNs fall on the AGN color track; in fact, some SMGs which contain AGNs fall on the starburst track. The interpretation of the positions of these galaxies in the $S_{24 \mu \mathrm{m}} / S_{8 \mu \mathrm{m}}$ versus $S_{8 \mu \mathrm{m}} / S_{4.5 \mu \mathrm{m}}$ diagram is unclear, but presumably the SMGs near the starburst track which contain AGNs are not AGN-dominated. The clustering of SMGs with intermediate starburst/AGN classifications near the starburst track is consistent with such a scenario. Another case in which the interpretation of the color-color diagram in especially unclear is that of the SMGs which fall between the starburst and AGN tracks. At least one confirmed AGN-dominated SMG, SMM J163650.43, lies in this region, which suggests that SMGs falling in this middle region are also AGN-dominated. Yet, the tracks of $S_{24} / S_{8.0}-S_{8.0} / S_{4.5}$ as a function of redshift for both NGC 6240 and the starburst nucleus of M82 lie in this middle region (Ivison et al. 2007), so SMGs falling in the middle could

\footnotetext{
11 Note that a lack of a previous AGN classification in this sample does not automatically indicate that it is a starburst, since for most of our sample the classifications are only available from rest-frame UV spectra, a region in which AGN spectral signatures are not always apparent.
} 
either be dominated by an obscured AGN or a starburst. Sajina et al. (2005) provide an alternate interpretation of the $S_{24} / S_{8.0}$ versus $S_{8.0} / S_{4.5}$ diagram which could explain why some SMGs fall between the starburst and AGN tracks. The simulations by Sajina et al. (2005), which are based on linear combinations of a mid-IR continuum spectrum from an AGN, a PAH emission spectrum, and a stellar continuum spectrum, suggest that the color-color diagram is an extinction sequence at high redshift in which both PAH-dominated and continuum-dominated galaxies are shifted to redder $S_{8.0 \mu \mathrm{m}} / S_{4.5 \mu \mathrm{m}}$ colors as the optical depth along the line of sight increases. In this scenario, some of the SMGs on the AGN track which have not been identified as AGNs through spectra, such as SMM J105200.22, could possibly be PAH-dominated sources with large extinction, while SMGs located between the starburst and AGN tracks may simply be PAH-dominated galaxies with moderate extinction. However, Sajina et al. (2005) point out that AGN-dominated sources do preferentially lie on the track defined by Mrk 231.

We conclude that the $S_{24 \mu \mathrm{m}} / S_{8.0 \mu \mathrm{m}}-S_{8.0 \mu \mathrm{m}} / S_{4.5 \mu \mathrm{m}}$ colorcolor diagram is a promising method to identify at least some AGN-dominated SMGs, and the diagram implies that most of our SMG samples are not dominated by a powerful, obscured AGN. However, given the uncertain classification of SMGs which fall between the AGN and starburst tracks, we caution that more investigation of the factors determining the $S_{24} / S_{8.0}$ and $S_{8.0} / S_{4.5}$ colors, especially in the form of mid-IR spectra, is needed before we can determine the efficiency with which the diagram separates AGN-dominated from starburst-dominated SMGs.

\subsubsection{Using a Near-IR Color-Color Diagram to Identify AGN-dominated SMGs}

Because redshift causes the IRAC filters to sample different rest wavelengths across our sample, efforts to identify AGNdominated galaxies from a single set of IRAC color criteria will be complicated and may fail. Using rest-frame colors for each SMG is a more direct way to compare. For galaxies at $z \sim 1.5-2.5$, the IRAC 3.6, 4.5, and $5.8 \mu \mathrm{m}$ bands sample the wavelength range of the broadband $J, H$, and $K$ filters; thus, with $K$-corrections we can derive rest-frame colors using $J, H$, and $K$ for individual galaxies and use a near-IR color-color diagram to identify AGN-dominated galaxies. Near-IR surveys of local galaxies (e.g., Joseph et al. 1984; Spinoglio et al. 1995) have shown that the total colors of active galaxies, dominated by both strong starbursts and AGNs, tend to stand out in, for example, a $J-H$ versus $H-K$ color-color diagram, as thermal emission from hot dust causes a strong color excess in $H-K$ as compared with "normal" galaxies which are dominated by stellar light in the near-IR. Scoville et al. (2000) show in a NICMOS F110W-F160W versus F160W-F222M color-color diagram (essentially $J-H$ versus $H-K$ ) that the nuclear colors of "warm" $\left(S_{25 \mu \mathrm{m}} / S_{60 \mu \mathrm{m}}>0.2\right)$, AGN-dominated ULIRGs stand out from "cold," starburst-dominated ULIRGs, and can be used to separate AGN-dominated galaxies from starburstdominated galaxies. Here, we construct a rest-frame $J-H$ versus $H-K$ color-color diagram for our SMG sample to identify AGN-dominated SMGs, which will both provide information on the AGN contribution of SMGs in the SSA 13 field which lacks MIPS data and serve as a check on the predictions of the $S_{24 \mu \mathrm{m}} / S_{8.0 \mu \mathrm{m}}-S_{8.0 \mu \mathrm{m}} / S_{4.5 \mu \mathrm{m}}$ diagram.

To derive the rest-frame $J-H$ and $H-K$ colors for our SMG sample, we have interpolated the $J H K$ fluxes of each SMG using the $J K$ photometry from Smail et al. (2004) and the IRAC photometry of this paper, using a simple linear interpolation, and converted the fluxes to $\mathrm{AB}$ magnitudes. For galaxies at redshifts $z>3$, the IRAC photometry does not adequately sample the $K$ band and we must extrapolate to determine a $K$-band flux, a considerable uncertainty in their colors; thus, the colors of these sources must be regarded with some caution. We see no evidence in the IRAC fluxes for strong contamination to the broadband fluxes from Paschen- $\alpha$ emission, and as it contributes nearly an order of magnitude less flux than $\mathrm{H} \alpha$ in case $\mathrm{B}$ recombination scenarios (Osterbrock 1989), we make no attempt to correct our interpolated colors for emission lines. We plot the $J-H$ colors versus $H-K$ in Figure 6. SMGs known to contain an AGN from optical, near-IR, or mid-IR spectra or X-ray detection are identified.

In Figure 6, we compare the rest-frame SMG colors to the nuclear colors (measured in a 1"1 aperture, which typically corresponds to $0.4-1 \mathrm{kpc}$ ) of the LIRG/ULIRG sample of Scoville et al. (2000), looking for SMGs which have integrated colors that could be nuclear-dominated. In general, we see that the SMGs tend to be bluer in both $J-H$ and $H-K$ than most of the LIRG/ULIRG nuclei, which suggests that the overall reddening of SMGs is not as severe as found in the nuclei of local (U)LIRGs. The LIRGs and ULIRGs whose nuclear colors do overlap with the majority of SMGs all have H IIregion-like nuclear spectra. Four SMGs display a clear $H-K$ excess, greater than that shown by PG QSOs and some of the "warm" LIRGs/ULIRGs, although these SMGs are usually bluer in $J-H$ than the local ULIRGs: SMM J030238.62, SMM J123600.15, SMM J123632.61, and SMM J131232.31. Scoville et al. (2000) find that the colors of such ULIRGs cannot be explained by reddened starlight alone, and the red $H-K$ color requires a strong contribution from hot $(600-1000 \mathrm{~K})$ dust emission at $K$ band. Based on their position in Figure 6, these SMGs show significant contributions to their rest-frame $K$ light from hot dust, and are likely to be dominated by a central AGN. An additional five SMGs, SMM J123635.59, SMM J131222.35, SMM J131215.27, SMM J141741.81, and SMM J163706.51, all of which are known to contain AGNs, also show evidence for a significant component of hot dust heated by a powerful AGN, though their $H-K$ colors are not as extreme. The $J-H$ versus $H-K$ diagram and $S_{24} / S_{8.0}$ versus $S_{8.0} / S_{4.5}$ diagram identify four potential AGN-dominated SMGs in common (although we cannot compare the identifications for the SSA 13 SMGs), which is encouraging consistency for the classifications of those four galaxies. Further investigation of the SMGs whose classifications differ between the methods will help determine if the methods have truly different selection functions.

We also show in Figure 6 the integrated colors of the LIRGs/ULIRGs in the Scoville et al. (2000) sample, measured through an $11^{\prime \prime} .4$ diameter aperture, which is a more appropriate comparison to the unresolved colors of the SMGs. In addition, we have plotted the total colors of "normal" galaxies (which do not have AGNs and which are not otherwise IR-luminous) from the $12 \mu \mathrm{m}$-selected sample of Spinoglio et al. (1995). First, we note that the integrated colors of the LIRGs/ULIRGs overlap much more with the SMG colors. Since Scoville et al. (2000) and Spinoglio et al. (1995) interpret the extended colors of the (U)LIRGs as evidence that the outer (non-nuclear) regions are stellar-dominated, we conclude that, in general, SMGs are not dominated by AGNs in the near-IR, but instead by stellar populations with ages $\sim 100$ s of Myr, behind moderate extinction $\left(A_{V} \sim\right.$ several mag). Also, the majority of SMGs and 


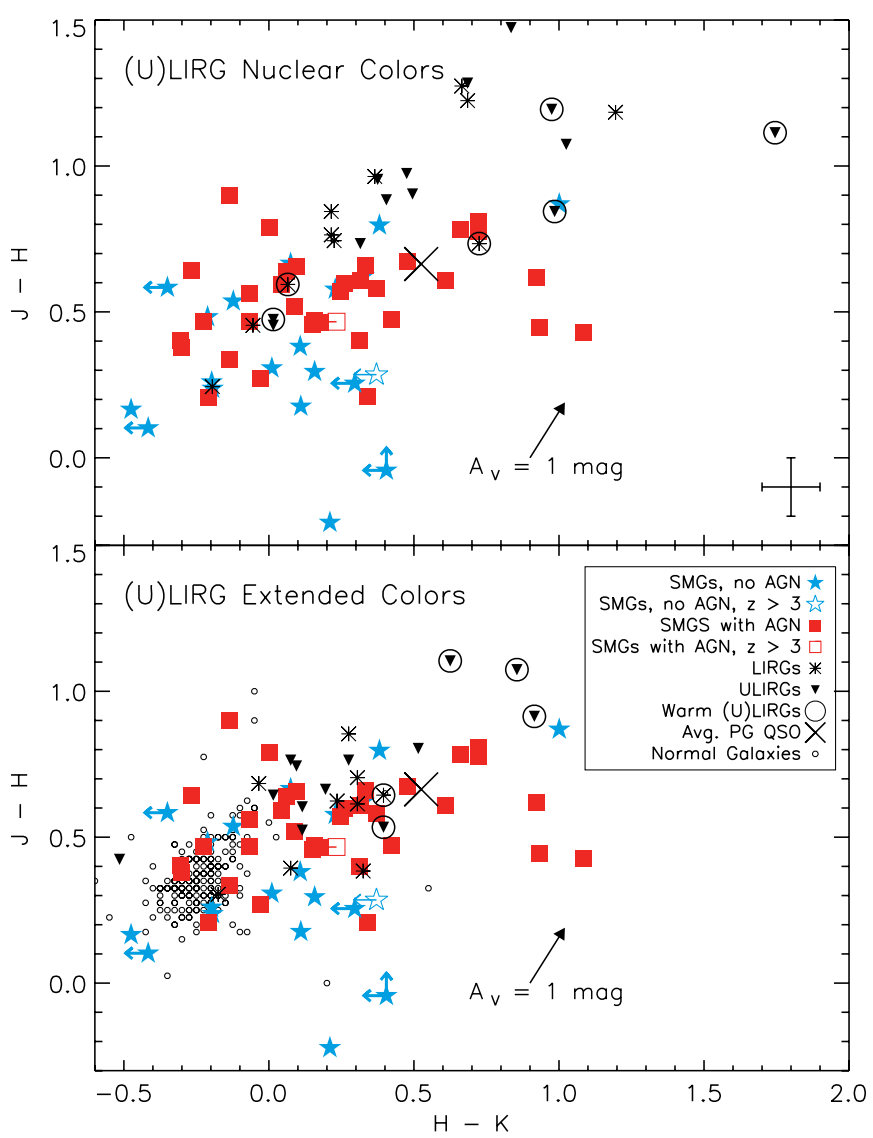

Figure 6. Rest-frame $J-H$ vs. $H-K$ color-color plot for SMGs. Symbols are as shown in inset in bottom panel; a typical error bar for SMG colors is shown in the lower right of the top panel. Galaxies for which the interpolation is less certain due to a nondetection in one or more IRAC bands are marked as upper limits. The reddening vector is shown in the lower right of each panel, calculated using the reddening law of Calzetti et al. (2000). Top panel: comparison of SMG colors to nuclear colors of local LIRGs and ULIRGs in the sample of Scoville et al. (2000). Bottom panel: comparison of SMG colors to extended-aperture colors of local LIRGs and ULIRGs from the sample of Scoville et al. (2000) and total colors of "normal" galaxies from the sample of Spinoglio et al. (1995). SMGs in our sample which were not detected in any IRAC band (5), or which were not covered by the IRAC imaging (4), have been excluded from the plot. Two additional SMGs in our sample have been excluded from the plot, since a combination of upper limits prevents a reliable estimate of one or more of the rest frame $J-H$ and $H-K$ colors. Eight SMGs have $H-K$ colors similar to AGN-dominated ULIRGs, while the majority of the SMGs appear instead to be dominated by stellar populations viewed through moderate extinction.

(A color version of this figure is available in the online journal.)

LIRGs/ULIRGs are redder in $H-K$ than the normal galaxies, which may be due to increased reddening on average, greater emission from warm dust in star-forming regions, or both.

The rest-frame near-IR color-color diagram is clearly useful in identifying powerful AGNs among SMGs; however, a redshift is required to determine the rest-frame colors. From the diagram, we identify at least four, possibly nine, SMGs which may be AGN-dominated. The color-color diagram and comparison of SMGs to LIRGs/ULIRGs thus suggest that the majority of SMGs are not AGN-dominated, even if the SMG is known to host an active nucleus, in agreement with Alexander et al. (2005a) and Menéndez-Delmestre et al. (2009).

The comparison of the rest-frame $J-H$ and $H-K$ colors of our sample of SMGs to the nuclear and extended colors of local LIRGs and ULIRGs also highlights an interesting point: the integrated near-IR colors of SMGs are not as extreme as the nuclei of ULIRGs or even LIRGs. The color differences between ULIRG nuclei and unresolved SMGs indicate lower line-of-sight extinction toward the starbursts in SMGs than in local ULIRGs, in agreement with the results of the midIR spectral analysis of SMGs in our sample in MenéndezDelmestre et al. (2009). The lower average extinction in SMGs provides additional evidence that star formation in SMGs occurs in a more extended, lower density environment than in ULIRGs, which has been suggested by studies of the radio morphology of SMGs. In high-resolution radio images, Chapman et al. (2004) and Biggs \& Ivison (2008) find that their SMG samples show extended radio emission to $\sim 1^{\prime \prime}$ in size $(\sim 8 \mathrm{kpc}$ at $z \sim 2$ ), which, using radio emission as a proxy for farIR emission, implies that the starbursting region in SMGs is larger than in ULIRGs and does not simply represent a scaledup nuclear starburst (although see Tacconi et al. 2008). The recent analysis of the IRS spectra of SMGs from our sample by Menéndez-Delmestre et al. (2009) has also suggested that the star formation activity is less concentrated than in local ULIRGs.

\subsubsection{Conclusions: Results of AGN Diagnostic Plots for SMGs}

We find from examination of the near and mid-IR colors of our sample of radio-selected SMGs that 9-13 out of 69 SMGs with Spitzer data (13\%-19\%) host AGNs whose emission dominates in the near- and mid-IR, some of which are not identified as such through their optical spectra. The fraction we find is similar to the $15 \%$ found in the mid-IR spectral study of Pope et al. (2008), and consistent with the results of Menéndez-Delmestre et al. (2009). Our result is slightly lower than the estimate of the overall fraction of SMGs hosting an AGN of 28\%-50\% found from an X-ray analysis by Alexander et al. (2005b); when we look at only the portion of our sample which is in common with Alexander et al. (2005b), we still obtain a lower AGN-dominated SMG fraction of $20 \%-25 \%$. When redshifts are available, as for our SMG sample, examination of the rest-frame $J-H$ and $H-K$ colors permits some distinction between starburstdominated and AGN-dominated galaxies. When redshifts are not available, the observed-frame $24 / 8.0 \mu \mathrm{m}$ and $8.0 / 4.5 \mu \mathrm{m}$ colors can identify AGN-dominated galaxies as well, although the interpretation of $24 / 8.0 \mu \mathrm{m}$ ratio is complicated by mid-IR spectral features.

\subsection{Comparison of SMG IRAC and MIPS Colors to Other High-z Galaxy Populations}

The various populations of high-redshift galaxies which are selected at different wavelengths (e.g., SMGs, Lyman break galaxies, $B z K \mathrm{~s}$, etc.) might seem to have little in common, since at the wavelengths at which they are selected the physical mechanisms responsible for their emission are different. Yet, all of the galaxy populations obviously have one characteristic in common which may prove to be the key to understanding how the populations relate to one another: stars. It is thus plausible that comparing the properties of the different high- $z$ populations at wavelengths where stellar emission dominates and the effects of dust are at a minimum (i.e., the IRAC bands) will be useful toward understanding the relationships between the different types of galaxies. With information from wavelengths where dust emission dominates (the MIPS bands), we may be able to glean information on AGNs content and/or the role of dust in the different galaxy types.

Here, we compare SMGs to several different types of high$z$ galaxies which span a range of mass, luminosity, and nuclear activity, hoping to shed some light on physical reasons 


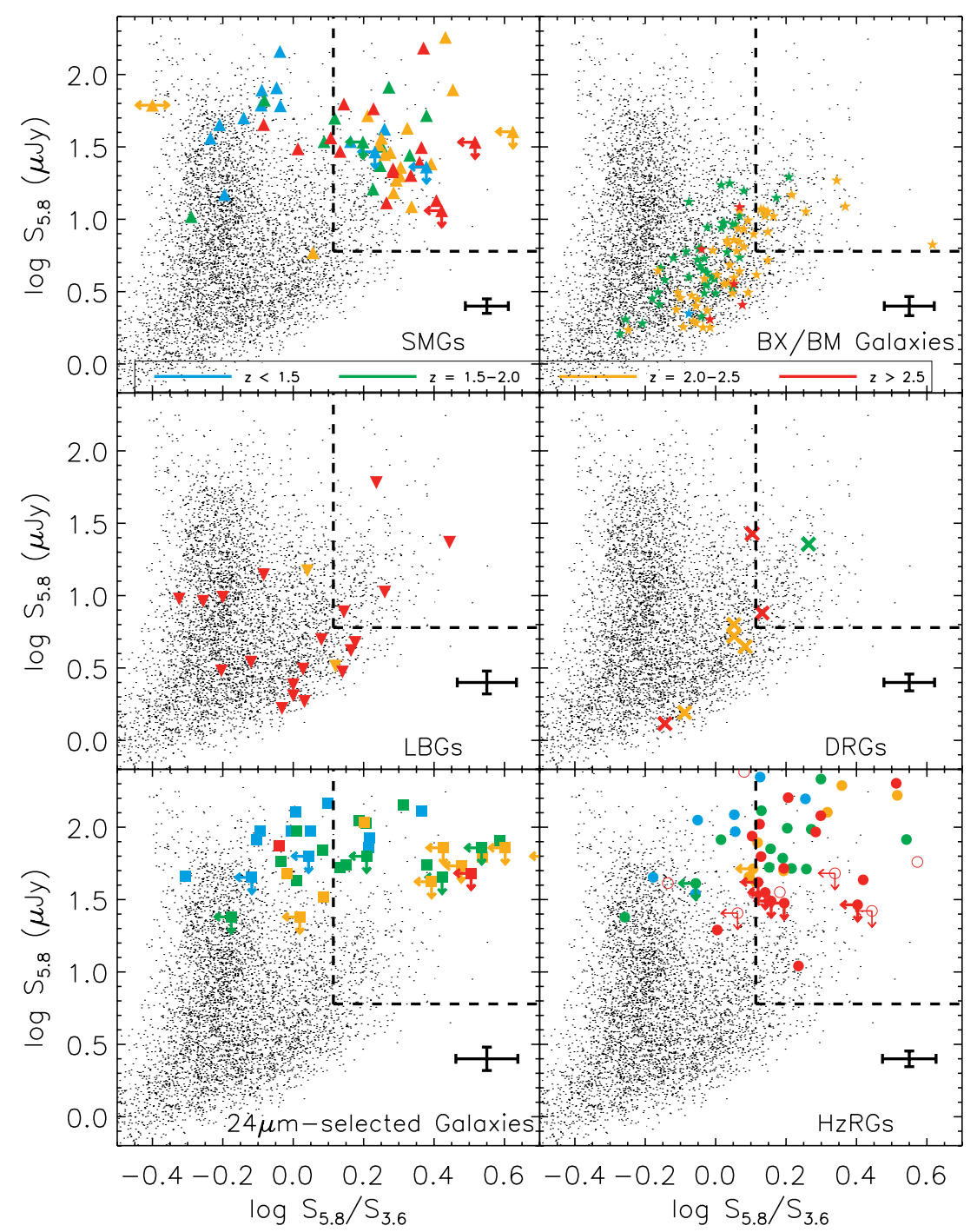

Figure 7. Comparison of $5.8 \mu \mathrm{m}$ vs. 5.8/3.6 $\mu \mathrm{m}$ color-magnitude diagrams for radio-detected SMGs in our sample, BX/BM galaxies, LBGs, DRGs, $24 \mu \mathrm{m}$-selected galaxies, and HzRGs. Field galaxies from GOODS-N are plotted for reference. The high- $z$ galaxy points are color-coded according to redshift. Open symbols indicate galaxies with $z>3.5$. Note that the majority of the LBGs are undetected at $5.8 \mu \mathrm{m}$ and their flux upper limits place them off the scale of the plot. Dashed lines indicate the region of the plot identified by Pope et al. (2006) as containing SMGs at $z>1.5$. A typical error bar for the data for each population is shown in the lower right of each panel. The IRAC colors of SMGs are significantly different from those of rest-frame UV-selected galaxies, unlike in observed-frame optical colors; the IRAC colors of SMGs are most similar to HzRGs. The IRAC fluxes and colors reflect that SMGs have higher stellar mass than UV-selected galaxies, and similar stellar mass to $24 \mu \mathrm{m}$-selected galaxies and HzRGs.

behind any observed differences between the galaxy types. Our comparison is not meant to be exhaustive; we merely wish to identify informative patterns. We prefer to use galaxies with spectroscopic redshifts for our comparison, so we construct our comparison sample from the catalog of $74 z \sim 3$ Lyman break galaxies (LBGs) and $212 z \sim 2 \mathrm{BX} / \mathrm{BM}$ galaxies of Reddy et al. (2006) in the GOODS-N field, galaxies which represent stellardominated star-forming galaxies with low obscuration; the sample of $4824 \mu \mathrm{m}$-selected galaxies from the Spitzer-xFLS presented by Yan et al. (2007, spectra) and Sajina et al. (2007a, photometry), which represent a different, more mid-IR-luminous, type of ultraluminous IR galaxy at similar redshifts to SMGs $(z \sim 2)$ and are thus an important comparison sample; and the sample of 69 powerful, high-z radio galaxies (HzRGs) from Seymour et al. (2007), which represent highly obscured, radio-loud AGNs with massive elliptical host galaxies at $z \sim 1-4$. In addition, we have separated out the galaxies in the sample of Reddy et al. (2006) which satisfy the distant red galaxy (DRG) criteria of Franx et al. (2003), which we treat separately as another com- parison set of galaxies; these DRGs also satisfy the BX/BM or LBG criterion, which means they likely are not representative of the entire DRG population. Unfortunately, large, representative samples of DRGs with spectroscopic redshifts are not readily available.

\subsubsection{Comparison of IRAC Colors}

We compare the different galaxy samples in Figure 7, using the same 5.8/3.6 $\mu \mathrm{m}-5.8 \mu \mathrm{m}$ color-magnitude diagram as shown in Figure 3. Each panel plots a different high-z galaxy type over the same sample of field galaxies from the GOODS$\mathrm{N}$ field used in previous plots (i.e., the galaxies which are not SMGs, LBGs, or BX/BM galaxies). Note that to remove the effects of the $K$-correction on galaxy colors, the same redshift slices of the different galaxy types should be compared. We also note that a majority of the LBGs are not detected at $5.8 \mu \mathrm{m}$, and the $3 \sigma$ upper limits on their fluxes place them just out of the diagram. We see immediately that the different high$z$ populations are distributed across the color-magnitude space, 


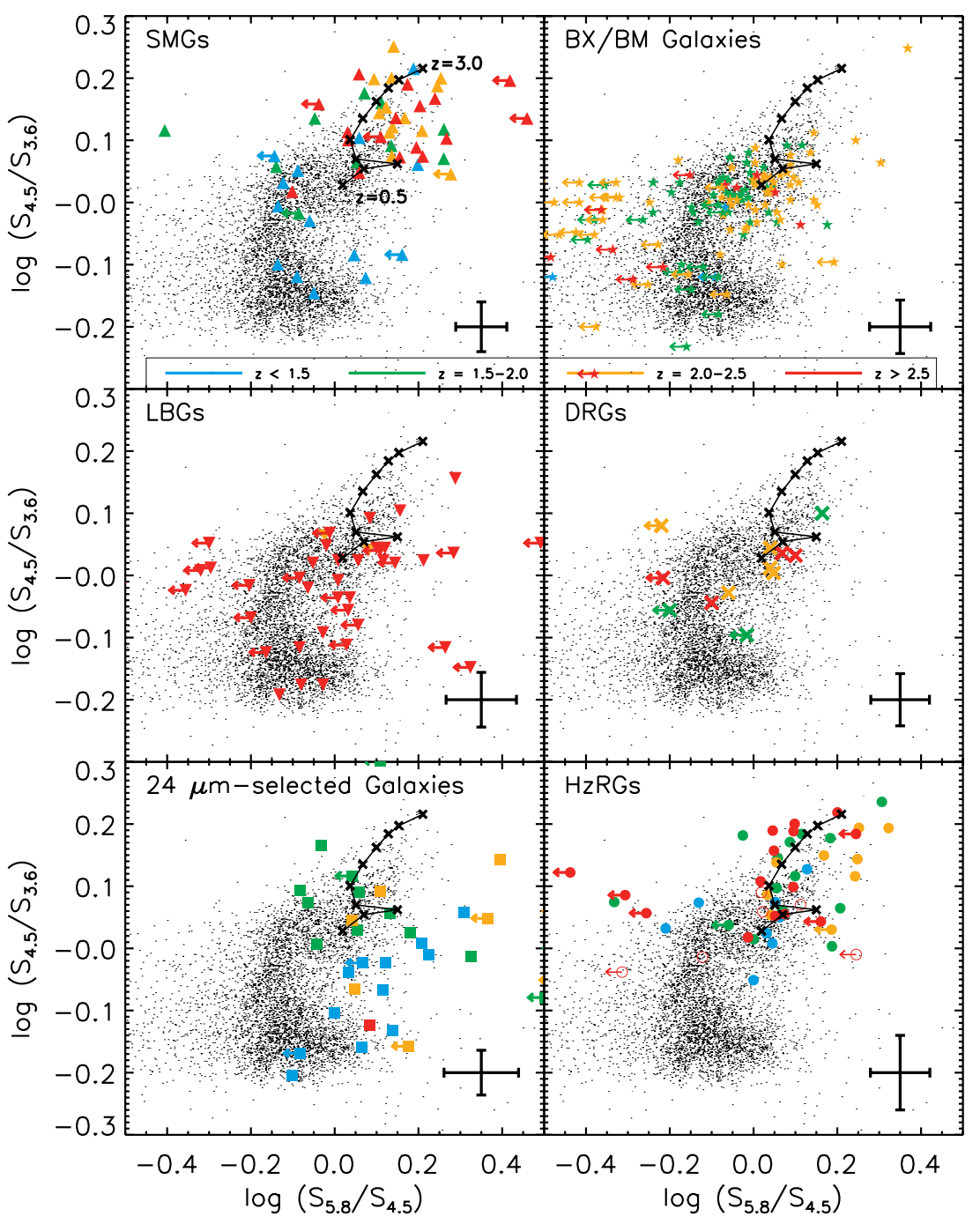

Figure 8. Comparison of 4.5/3.6 $\mu \mathrm{m}$ vs. 5.8/4.5 $\mu \mathrm{m}$ color-color diagrams comparing SMGs in our sample to field galaxies and samples of BX/BM galaxies, LBGs, DRGs, $24 \mu \mathrm{m}$-selected galaxies, and HzRGs. Symbols are as in Figure 7. The solid line represents the redshifted 4.5/3.6 $\mu \mathrm{m}$ and 5.8/4.5 $\mu \mathrm{m}$ colors of the nuclear region of M82 at redshifts $z=0.50,0.70,0.75,0.80,1.00,1.25,1.50,1.75,2.00$, and 3.00. A typical error bar for the data for each population is shown in the lower right of each panel.

but the individual galaxy types are not distinct enough to be able to cleanly separate each one from the others. The SMGs, HzRGs, and $24 \mu \mathrm{m}$-selected galaxies are clearly brighter on average at $5.8 \mu \mathrm{m}$ and redder in $S_{5.8} / S_{3.6}$ than the optical and UV-selected galaxies at similar redshifts, though the $z \sim 1.5-2.5 \mathrm{HzRGs}$ may be slightly brighter than their SMG contemporaries. The $5.8 \mu \mathrm{m}$ fluxes of the SMGs and UV/optically selected galaxies and the more prominent stellar bumps in their near-IR SED are consistent with SMGs having larger stellar masses overall (see Borys et al. 2005; Paper II). The color-magnitude diagram suggests that SMGs have similar stellar mass to HzRGs, which is borne out in fitting the near-IR SEDs of the populations (Seymour et al. 2007; Paper II), and that $24 \mu \mathrm{m}$-selected galaxies have similar stellar masses as well. The presence of hot dust heated by a powerful AGN will complicate the interpretation of the color-magnitude plot; however, in the case of SMGs, our analysis of the rest-frame $J-H$ and $H-K$ colors in Section 4.3.3 indicates that most SMGs are not dominated by a powerful AGN in the rest-frame near-IR. We note that the colors of the UV/ optically selected galaxies, which generally overlap the faint field galaxy (non-SMG, non-BX/BM, non-LBG) population, are clearly bluer than SMGs of similar redshifts and there is little overlap between UV-selected and submm-selected galaxies. This strongly contrasts with their observed-frame optical colors: C05 note that $65 \%$ of the SMG sample with deep $U$-band data are blue enough to satisfy the $\mathrm{BX} / \mathrm{BM}$ selection criteria, and only $30 \%$ of the SMGs were too faint to be classified as LBGs or BX/BM galaxies. The color differences between SMGs and $\mathrm{LBG} / \mathrm{BX} / \mathrm{BM}$ samples in the rest-frame UV and near-IR further underscores the differences in stellar mass and dust extinction between them.

In Figure 8, we show a 4.5/3.6 $\mu \mathrm{m}$ versus 5.8/4.5 $\mu \mathrm{m}$ color-color diagram for each of the different samples of high- $z$ galaxies. Galaxies from all of the samples must have detections in the 3.6 and $4.5 \mu \mathrm{m}$ IRAC bands to be included in this plot; we lose only $\sim 10 \%$ of the galaxies from each sample through this requirement, and thus our results will still be representative of the different populations. For comparison, we have added the track of $S_{4.5} / S_{3.6}$ versus $S_{5.8} / S_{4.5}$ as a function of redshift for $0.5<z<3.0$ for the nuclear region of the starburst galaxy M82, using the SED fit of Siebenmorgen \& Krügel (2007) to the observed data for the nuclear region of M82. In the figure, we immediately notice that the distribution of $z>1.5 \mathrm{SMGs}$ in the color space once again most resembles 
that of the powerful high- $z$ radio galaxies. Both the SMGs and HzRGs are frequently found to be red in both $S_{4.5} / S_{3.6}$ and $S_{5.8} / S_{4.5}$, which suggests that the $z>1.5$ SMGs are dominated by powerful AGNs like the HzRGs. However, Seymour et al. (2007) find that half of their sample of HzRGs observed by both IRAC and MIPS are dominated by starlight in the rest-frame near-IR, while for another $20 \%$ of their sample $60 \%-90 \%$ of the rest-frame near-IR luminosity can be attributed to starlight. This contradictory-seeming situation (stellar light dominating a powerful AGN) can be attributed to the orientation of the central AGN under AGN unification scenarios (e.g., Antonucci 1993). Thus, the near-IR colors of the radio-loud galaxies may not be entirely reflecting an active nucleus. We note that both SMGs and HzRGs have IRAC colors similar to the redshifted colors of the stellar-dominated M82, so the color similarity between SMGs and radio-loud galaxies may indicate instead that the two types have similar contributions from hot dust heated by star formation as M82 does, or that SMGs and HzRGs have similarly massive, reddened stellar populations.

On the other hand, if SMGs are stellar-dominated, why do their $S_{4.5} / S_{3.6}$ and $S_{5.8} / S_{4.5}$ colors show only small overlap with BX/BM galaxies and LBGs of similar redshift? There are two possible answers to this puzzle: SMGs are more reddened by dust, or SMGs have a larger AGN continuum contribution than the UV-selected galaxies. Our rest-frame $(J-H, H-K)$ color analysis in Section 4.3.3 suggests that the AGN contribution to the rest-frame near-IR light of SMGs does not dominate over the stars, so once again we conclude that the difference in near-IR colors between SMGs and UV-selected galaxies can be attributed to higher extinction in SMGs. Also, there are $\sim 20 \mathrm{BX} / \mathrm{BM}$ galaxies and LBGs whose IRAC colors do overlap with the $z>1.5 \mathrm{SMGs}$, perhaps indicating that the red IRAC colors can be consistent with stellar-dominated galaxies. These relatively red UV-selected galaxies probably represent the IR-luminous LBGs (LBGs detected at $24 \mu \mathrm{m}$ ) suggested by Huang et al. (2005) as possible bridges between LBG and SMG population.

The $24 \mu \mathrm{m}$-selected galaxy sample does not appear to overlap a great deal with SMGs or any of the other galaxy populations represented in Figure 8 in the $S_{4.5} / S_{3.6}-S_{5.8} / S_{4.5}$ color space, particularly those $24 \mu \mathrm{m}$-selected galaxies at $z<1.5$. The $z<1.5$ galaxies are generally redder in $S_{5.8} / S_{4.5}$ than SMGs of similar redshifts. Because the 4.5 and $5.8 \mu \mathrm{m}$ IRAC bands sample well to the red of the $1.6 \mu \mathrm{m}$ bump for galaxies at these redshifts, the redder colors of the $24 \mu \mathrm{m}$-selected galaxies indicate larger contributions from hot dust continuum in the restframe near-IR than found in SMGs. The hot dust continuum could arise from emission from powerful, obscured AGNs or compact starburst; both interpretations could be supported by the IRS spectra of the $24 \mu \mathrm{m}$-selected galaxies, which show contributions from strong continuum and strong PAH features (Sajina et al. 2007a).

\subsubsection{Comparison of $24 \mu \mathrm{m} / I R A C$ Colors}

Next, we turn our attention to the $24 \mu \mathrm{m} /$ IRAC colors of the different high- $z$ galaxy populations. In Figure 9, we plot the $S_{24} / S_{8.0}-S_{8.0} / S_{4.5}$ color-color diagram from Ivison et al. (2004), comparing SMGs, BX/BM galaxies, $24 \mu \mathrm{m}$-selected galaxies, and high- $z$ radio galaxies, noting that only one-third of the high$z$ radio galaxies have $24 \mu \mathrm{m}$ data. While we have overplotted the color tracks of Arp 220 and Mrk 231 as in Figure 5, we are not explicitly looking to separate AGNs from starbursts in this plot; the tracks are merely reference points. The different types

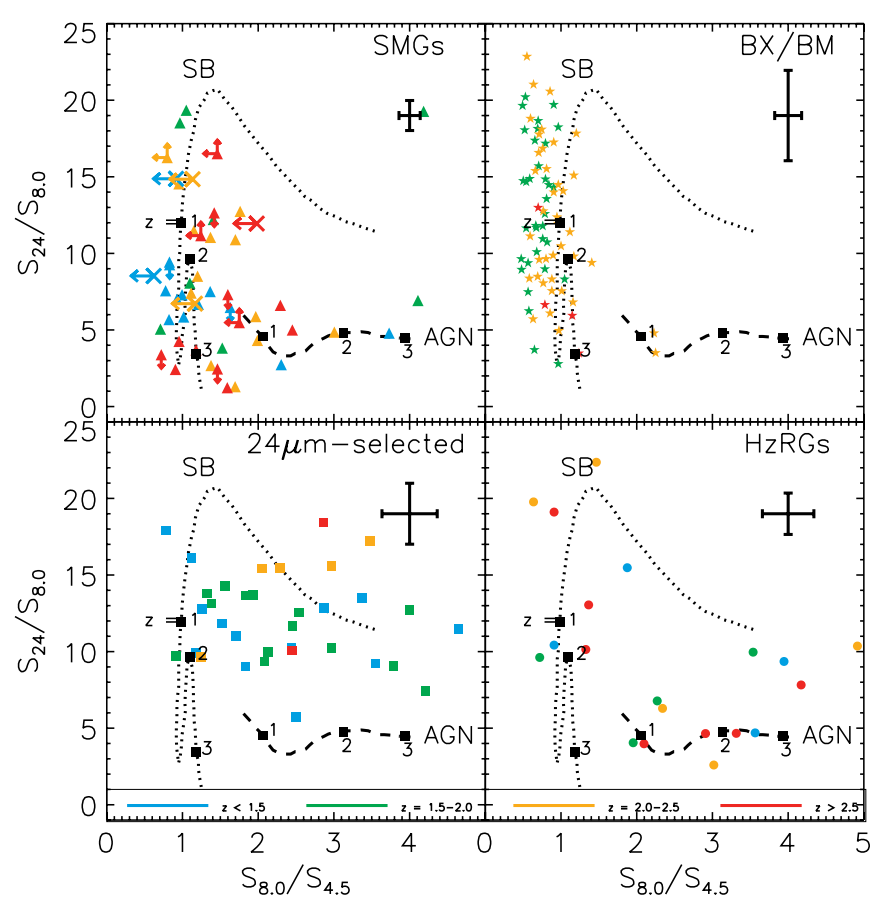

Figure 9. Comparison of $S_{24} / S_{8.0}$ vs. $S_{8.0} / S_{4.5}$ color-color diagrams for BX/ BM galaxies, $24 \mu \mathrm{m}$-selected galaxies, and HzRGs. The points are color coded by redshift as in Figure 7. The dotted and dashed lines are as in Figure 5. A typical error bar for the data for each population is shown in the upper right of each panel. From this diagram, we suggest that BX/BM galaxies, SMGs, $24 \mu \mathrm{m}$ selected galaxies, and HzRGs represent a sequence in which the contribution of AGN luminosity relative to star formation luminosity increases.

of galaxies have somewhat different distributions in color space. Unlike the other galaxy populations shown in the plot, the BX/ BM galaxies with $24 \mu \mathrm{m}$ detections almost exclusively fall near the color-color track of Arp 220. The HzRGs display a rather similar distribution in color space to that of the SMGs; however, the $24 \mu \mathrm{m}$-selected galaxies appear to be totally different from the SMGs and HzRGs in the $S_{24} / S_{8.0}-S_{8.0} / S_{4.5}$ color space. The $24 \mu \mathrm{m}$ galaxies cover the same range in $S_{8.0} / S_{4.5}$, yet their $S_{24} / S_{8.0}$ flux ratio at a given value of $S_{8.0} / S_{4.5}$ is generally larger for galaxies of all redshifts.

The higher $S_{24} / S_{8.0}$ ratios of $24 \mu \mathrm{m}$-selected galaxies over SMGs are most likely the result of the $24 \mu \mathrm{m}$-selected galaxies being brighter at $24 \mu \mathrm{m}$ (the median $24 \mu \mathrm{m}$ flux and $1 \sigma$ scatter is $\left\langle S_{24}\right\rangle=1300 \pm 770 \mu \mathrm{Jy}$ for the $24 \mu \mathrm{m}$-selected galaxy sample, as compared with $\left\langle S_{24}\right\rangle=231 \pm 735 \mu \mathrm{Jy}$ for our SMG sample). By looking at the mid-IR spectral characteristics for the $24 \mu \mathrm{m}$ selected galaxies, we can determine why they are brighter than SMGs at $24 \mu \mathrm{m}$. The increase in luminosity is not due to more emission in the PAH features compared with SMGs: the $24 \mu \mathrm{m}$ selected galaxies display $7.7 \mu \mathrm{m}$ PAH features which have the same range of luminosity as the $7.7 \mu \mathrm{m}$ PAH emission in SMGs (Menéndez-Delmestre et al. 2009). Thus, the $24 \mu \mathrm{m}$-selected galaxies must have more luminous dust continua, potentially due to a higher AGN contribution.

In comparing the different galaxy populations in the $S_{24} / S_{8.0}$ $S_{8.0} / S_{4.5}$ color-color diagram, the suggestion of Sajina et al. (2005) that the trends in this diagram might be a function of extinction at high redshift seems plausible. We see that the BX/ BM galaxies, which are known to have low extinction since we select them from their rest-frame UV light, lie consistently on the far left of the plot. The $24 \mu \mathrm{m}$-selected galaxies, which must contain significant dust, move toward the right of the plot and scatter significantly more. SMGs could be seen as having 
varying quantities of extinction since they are observed all over the plot. However, a significant fraction of the SMGs lie in the same region of color space as the $\mathrm{BX} / \mathrm{BM}$ galaxies, which by the extinction interpretation would indicate they have low extinction; we know from the IRAC color diagrams, though, that SMGs clearly have larger extinction than the majority of UV-selected galaxies.

We suggest instead that stellar-dominated galaxies at $z \gtrsim 2$ will have $S_{8.0} / S_{4.5}<1.5$, like the majority of BX/BM galaxies in the color-color diagram, but will show a range in $S_{24} / S_{8.0}$ which depends on their PAH feature luminosity and specific star formation rate. $z \sim 2$ galaxies with significant contributions from AGNs will have $S_{8.0} / S_{4.5}>1.5$ as the hot dust begins to dominate the $8.0 \mu \mathrm{m}$ band flux, while the $S_{24} / S_{8.0}$ ratio for these galaxies will vary according to the ratio of PAH luminosity to continuum luminosity, which again will depend on their SFR. AGN-dominated galaxies with featureless mid-IR continua and low SFR will have $S_{8.0} / S_{4.5}>1.5$ and low $S_{24} / S_{8.0}$, but galaxies in which the AGN dominates though significant star formation is ongoing will have higher $S_{24} / S_{8.0}$, increasing with $\mathrm{PAH}$ feature luminosity and starburst luminosity-AGN luminosity ratio. Thus, the $S_{8.0} / S_{4.5}$ ratio becomes the important energetic AGN indicator. Under such an interpretation of the $S_{24} / S_{8.0}-$ $S_{8.0} / S_{4.5}$ color-color diagram, the BX/BM galaxies, SMGs, $24 \mu \mathrm{m}$-selected galaxies, and HzRGs may be construed as a sequence in which the importance of AGN luminosity relative to star formation luminosity shifts. BX/BM galaxies represent the dominance of star formation, with the vast majority of galaxies at $S_{8.0} / S_{4.5}<1.5$. SMGs might represent the beginning of AGN contribution to mid-IR luminosity but are still generally dominated by star formation, since most of the galaxies have $S_{8.0} / S_{4.5}<1.5$ but a few have $S_{8.0} / S_{4.5}>1.5$. $24 \mu \mathrm{m}$ selected galaxies could represent a truly intermediate phase in which the star formation component and AGN component both contribute significantly to the mid-IR luminosity, since most of the galaxies have $S_{8.0} / S_{4.5}>1.5$ but have significant PAH luminosity causing high $S_{24} / S_{8.0}$. HzRGs, then, could represent a phase which is almost fully AGN-dominated, where for most of the galaxies $\mathrm{PAH}$ features are no longer significant in the mid-IR spectra, causing generally low $S_{24} / S_{8.0}$. Note that we are not necessarily suggesting that this is an evolutionary sequence because the space densities of the different galaxy types do not match, but the small fraction of SMGs which share the mid-IR properties of $24 \mu \mathrm{m}$-selected galaxies (e.g., SMM J123600.15) and the $24 \mu \mathrm{m}$-selected galaxies which are radio-loud and show radio jets (Sajina et al. 2007b) suggest that transition objects exist. Detailed analysis of mid-IR spectra for a representative sample of HzRGs will be useful to evaluate our proposed sequence of AGN contribution to overall luminosity; currently only two HzRGs have mid-IR spectra published (Seymour et al. 2008).

\section{SUMMARY AND CONCLUSIONS}

SMGs represent a population of high-redshift, ultraluminous galaxies, thought to be powered by very high levels of obscured star formation, and suggested as the progenitors of some of the most massive galaxies observed in the local universe. Here we have presented mid-IR imaging obtained with the IRAC and MIPS instruments on board the Spitzer Space Telescope of 73 radio-detected SMGs with spectroscopic redshifts from the survey of Chapman et al. (2005), the largest study using Spitzer of SMGs with spectroscopic redshifts to date. Our observations are consistent with the picture of SMGs as predominantly high redshift, highly reddened star-forming galaxies. Our main conclusions are the following.

1. SMGs are redder than field galaxies in IRAC colors, which is attributed to their redshifted $1.6 \mu \mathrm{m}$ stellar bump, strong extinction, and high stellar mass. They are also redder in their rest-frame $J H K$ colors than local quiescent galaxies, suggestive of a component of dust heated by star formation which quiescent galaxies lack, or higher reddening.

2. A rest-frame $J-H$ versus $H-K$ color-color diagram and a $S_{24} / S_{8.0}$ versus $S_{8.0} / S_{4.5}$ color-color diagram indicate that $\sim 80 \%$ of the SMGs in our sample are starburst-dominated in the near/mid-IR; the remaining $20 \%$ contain significant AGN contributions.

3. The rest-frame near-IR emission of SMGs is not consistent with a scaled-up version of the compact starburst nuclei found in local ULIRGs. Rather, SMGs appear less reddened, which is consistent with other evidence that star formation is occurring over a large region in these galaxies.

We have also compared the IRAC and MIPS colors of SMGs to those of high-redshift UV-selected galaxies, $24 \mu \mathrm{m}$-selected galaxies, and powerful high- $z$ radio galaxies, in an attempt to determine possible relationships between the populations selected at different wavelengths. We conclude the following from our comparisons.

1. The IRAC fluxes and colors (rest-frame near-IR) of SMGs are brighter and redder than those of UV-selected galaxies, consistent with SMGs having larger stellar mass and higher reddening, as well as larger AGN contributions.

2. The IRAC colors of SMGs are most similar to those of high$z$ radio galaxies that contain powerful, obscured AGNs in massive elliptical host galaxies. However, the colors of the SMGs and HzRGs are still consistent with being stellardominated and highly obscured, indicating that SMGs and HzRGs have similar stellar populations.

3. A comparison of the $S_{24} / S_{8.0}$ versus $S_{8.0} / S_{4.5}$ color-color diagrams for the different galaxy populations suggests that the UV-selected galaxies, SMGs, $24 \mu \mathrm{m}$-selected galaxies, and HzRGs form a sequence in which the AGN contribution to the bolometric luminosity increasingly dominates over the contribution of star formation.

Wide-field surveys utilizing the next generation of submm instruments, including SCUBA-2, Herschel, and CCAT, will reveal large new samples of SMGs which will require considerable quantities of time for follow-up to determine counterparts, redshifts, and AGN contribution. We have therefore looked for trends with redshift and examined methods suggested in the literature for IR SMG counterpart identification and AGN identification with our Spitzer data which can be used effectively on large samples of SMGs. We reach the following conclusions from our efforts.

1. The $S_{5.8}$ versus $S_{5.8} / S_{3.6}$ color-magnitude diagram suggested by Pope et al. (2006) is generally successful in separating out $z>1.5$ SMGs from lower redshift galaxies, selecting out $83 \%$ of the $z>1.5$ SMGs in our sample with a $4 \%$ low- $z$ galaxy contamination rate.

2. Color-magnitude and color-color cuts proposed by Pope et al. (2006) and Yun et al. (2008) for the identification of SMG counterparts successfully identify the single radio counterpart for the SMGs in our sample in an $8^{\prime \prime}$ matching radius for $53 \%$ and $62 \%$ of our radio-detected SMG sample, respectively. However, for $18 \%$ and $35 \%$ of our sample, 
the selection criteria of Pope et al. (2006) and Yun et al. (2008), respectively, produce ambiguous counterparts. The IRAC selection of SMG counterparts is complicated by many SMGs having IRAC fluxes and colors similar to more quiescent field galaxies, especially at $z<1.5$.

3. Rest-frame $J H K$ colors and the $S_{24} / S_{8.0}$ versus $S_{8.0} / S_{4.5}$ color-color diagram proposed by Ivison et al. (2004) both appear to be promising methods to select out SMGs which are bolometrically dominated by AGN. The $S_{24} / S_{8.0}$ versus $S_{8.0} / S_{4.5}$ diagram is the most likely to be useful in future surveys of SMGs since it does not require knowledge of a galaxy's redshift.

Our results from examination of the Spitzer continuum fluxes and colors of SMGs are suggestive of trends in their stellar mass, IR SED properties, and IR luminosity. We investigate the stellar characteristics and mass of SMGs explicitly through analysis of the rest-frame UV-near-IR SEDs of our sample of Spitzer-observed SMGs in L. J. Hainline et al. (2009a, in preparation). In L. J. Hainline et al. (2009b, in preparation), we explore the mid-far-IR SED properties of our sample with MIPS observations and the implications the MIPS data have for the dust temperatures and IR luminosities of these galaxies through SED model fitting.

We thank the anonymous referee for comments and suggestions which improved the manuscript. We also thank R. Chary, M. Salvato, and C. Borys for their very helpful advice in reducing IRAC and MIPS data. I.R.S. and D.M.A. acknowledge support from the Royal Society. The Spitzer Space Telescope is operated by the Jet Propulsion Laboratory at the California Institute of Technology, under contract with NASA.

Facilities: Spitzer ()

\section{APPENDIX A}

\section{IRAC DATA PROCESSING}

\section{A.1. Artifact Removal and Mosaicking}

We began reduction of the IRAC data for all of the SMG fields except the GOODS-N field with the basic calibrated data (BCD) images produced by version 14.0 (v14.0) of the Spitzer Science Center (SSC)'s IRAC Data Reduction Pipeline. We have excluded from all further processing the first two frames in each imaging sequence in each IRAC band (DCENUMs 0 and 1) since these frames have shorter times between data frames and cannot be calibrated correctly because the DC levels in the instrument change as a function of the time elapsed between frames.

Pipeline v14.0 processing includes dark subtraction, detector linearization, flat fielding, cosmic ray detection, and flux calibration to units of $\mathrm{MJy} \mathrm{sr}^{-1}$. However, pipeline v14.0 did not correct image artifacts such as column pull-down, multiplexer bleed ("muxbleed"), persistent images, scattered light from sources out of the field of view of the detector, and ghost images caused by internal reflections within the filters. In addition, the pipeline does not correct residual bias patterns caused by the use of in-place repeats, which is most prominent in channel 3.

Many of our BCD images suffered from at least some of these possible artifacts, and since we expected our target objects to be at the faintest flux levels in the images, it was necessary to remove the artifacts to improve our detection sensitivity and prevent the association of a target source with spurious detections. To accomplish this, we used the IDL IRAC Artifact Mitigation scripts ${ }^{12}$ written by S. Carey at the SSC to remove column pull-down and muxbleed from all of our images. We used the Muxstripe Corrector ${ }^{13}$ written by J. Surace at the SSC, to remove the pinstriping associated with muxbleed (iterating the script for the number of 2MASS stars in the image). For the SMG fields in which in-place repeats were used in the data-taking process (CFRS-03h, ELAIS-N2, and SSA 22), we modified the Repeat-Delta-Dark script written in IDL by S. Carey ${ }^{14}$ to remove the residual bias patterns from channel three BCD images. Image artifacts caused by stray light and persistent images were masked using the Stray Light Masker written by M. Lacy and R. Arendt at the SSC. ${ }^{15}$

After artifact mitigation and before constructing mosaics for each field, we constructed "superflats" from our own data to remove higher-order detector response effects and some persistent images. The superflats were made for each field and each channel by masking out 2MASS sources brighter than $K \sim 13$ in individual BCDs and then taking the median of the masked images. The normalized superflats were then applied to all BCDs.

We created mosaic images for each IRAC channel for each SMG field using the MOPEX software package (Makovoz \& Marleau 2005). After constructing a common coordinate and geometric frame for all the artifact-corrected BCD images in all of the IRAC channels, we carried out absolute pointing refinement of the individual BCD frames, aligning each frame to the 2MASS system using bright $(K<14)$ MASS point sources in each image, to facilitate the co-addition of overlapping images in the mosaic. Pointing refinement was generally successful in channels 1 and 2; however, in channels 3 and 4, there were not always enough bright 2MASS stars visible for the pointing solution to be derived. Thus, if the absolute pointing refinement failed, we performed relative pointing refinement. Afterward, we equalized the background of individual median skysubtracted BCD images, interpolated the background-matched frames into the common grid using the "drizzling" interpolation method with a drizzling factor of 0.8 , and corrected the images for optical distortion. To improve the cosmetic quality of the mosaics, we identified and masked deviant pixels (e.g., due to cosmic ray hits, bad array pixels, or image artifacts) with spatial and temporal filtering. The combination of outlier rejection based on spatial and temporal filtering proved more effective than either method alone, since most of the data sets had variable coverage over the imaged fields and lacked the high redundancy required for temporal filtering only. Finally, the interpolated science images were co-added using the masks of rejected pixels, weighting pixels by exposure time. The final mosaics have pixels of size 0!' 6 in all fields.

For the GOODS-N field, the already-reduced and mosaicked IRAC images were downloaded from the GOODS Spitzer Legacy Project's Web site. ${ }^{16}$ The processing and mosaicking of these data are described in M. Dickinson et al. (2009, in preparation), and is similar to that performed for the rest of the SMG fields. The final mosaic also has 0'.6 pixels, and its astrometry has been tied to the Hubble Space TelescopeAdvanced Camera for Surveys (HST-ACS) and VLA images of the GOODS-N field.

\footnotetext{
${ }^{12} \mathrm{http} / / /$ spider.ipac.caltech.edu/staff/carey/irac-artifacts

$13 \mathrm{http}: / / \mathrm{ssc}$.spitzer.caltech.edu/irac/automuxstripe

$14 \mathrm{http}: / /$ ssc.spitzer.caltech.edu/irac/deltadark

15 http://ssc.spitzer.caltech.edu/irac/straylight

16 http://data.spitzer.caltech.edu/popular/goods/
} 


\section{A.2. IRAC Source Extraction}

We detected and extracted fluxes for the SMGs in each field in each IRAC channel using SExtractor version 2.5.0 (Bertin $\&$ Arnouts 1996). We measure fluxes through $4^{\prime \prime}$ diameter apertures in each channel, centered on the IRAC counterpart of the SMG, which was identified through the position of the SMG's radio counterpart, not from the SCUBA position. We then apply aperture corrections to a $12^{\prime \prime}$ radius aperture, the size of which was obtained by a curve-of-growth analysis of isolated 2MASS point sources in each field to determine the radius enclosing the total flux in all channels. The $12^{\prime \prime}$ radius aperture also matches the IRAC absolute calibration aperture.

For undetected SMGs, we list $3 \sigma$ upper limits calculated from the standard deviation of the flux measured in empty apertures near the position of the galaxy in question. By measuring the rms of empty apertures near the object's radio position the upper limits we calculate take into account the nonuniform coverage within the imaged fields and reflect more accurately the depth of imaging at the object's position.

We expect the measured source fluxes to be accurate to better than $10 \%$ for IRAC channels 1 and 2, including absolute flux calibration errors (typically 3\%), aperture correction errors (typically $2 \%-3 \%$ ), errors due to uncorrected IRAC data artifacts (e.g., pixel phase correction in channel 1), and errors due to color corrections (usually less than $1 \%$ for sources with spectra of shape $F_{v} \propto v^{-2}$ ). In channels 3 and 4 , we expect the fluxes to be accurate to $\sim 10 \%$ since errors in the aperture corrections tend to be larger at the longer wavelengths. The positions of the detected sources should be accurate to $\sim 0.5$ in channels 1 and 2 since the absolute pointing has been aligned to the accuracy of 2MASS $\left(\sim 0^{\prime \prime} .15\right)$ with an rms of typically 0.3 . We expect that the dominant position error in these channels comes from centroiding due to the under-sampled PSF of IRAC. In channels 3 and 4 , where absolute pointing refinement was unsuccessful, the source position errors have a more significant contribution from the blind pointing error of IRAC $\left(\sim 0^{\prime \prime} .5\right)$, so we conservatively assume that the positions are accurate to $\sim 1^{\prime \prime}$.

\section{A.3. Flux Comparison to Literature Measurements}

We have compared our IRAC photometry to previously published values (Egami et al. 2004; Borys et al. 2005; Pope et al. 2006; Ashby et al. 2006) for cases in which we agree on the IRAC counterpart selected by the other studies. Our photometry in all four IRAC bands agrees with both Borys et al. (2005) and Pope et al. (2006) to within 5\% and with Ashby et al. (2006) to within 10\%-15\%; however, our fluxes are consistently 10\%-30\% higher than those of Egami et al. (2004), depending on the wavelength being compared. We suspect that the large differences between our photometry and that of Egami et al. (2004) arise because of the different ways we measure the sources. We have performed aperture photometry and applied wavelength-dependent aperture corrections, whereas Egami et al. (2004) use PSF fitting.

\section{APPENDIX B}

\section{MIPS-24 $\mu \mathrm{m}$ DATA PROCESSING}

\section{B.1. Data Processing and Mosaicking}

For all of the imaged SMG fields except the HDF/GOODS$\mathrm{N}$ field, we began reduction of the $24 \mu \mathrm{m}$ MIPS data with the BCD images produced by version 14.4.0 (v14.4) of the SSC's MIPS data reduction pipeline. We excluded the first frame in each imaging sequence (those with DCENUM =0) since these frames have a shorter exposure time than the rest.

Pipeline v14.4 processing includes data linearization, removal of time-dependent responsivity variations, dark subtraction, flat-fielding, flagging of pixels affected by cosmic rays and saturation, and flux calibration to units of $\mathrm{MJy} \mathrm{sr}^{-1}$ (Gordon et al. 2005). To mitigate the effects of image artifacts and improve the sensitivity of the BCD images, we applied the selfcalibration procedure described in the MIPS Data Handbook, v. 3.2.1 (Spitzer Science Center 2006). In this process, for each Astronomical Observing Request (AOR) a normalized median of all the images, excluding very bright pixels, is constructed from primary array BCDs (PRIMEARR = 1). The individual $\mathrm{BCDs}$ in that AOR are subsequently divided by this normalized median image.

The median-calibrated primary array BCD images from all AORs available for a given SMG field were next combined into a single $24 \mu \mathrm{m}$ mosaic using the MOPEX software package. In a similar procedure to that used to create the mosaics of IRAC BCD images, we created a common coordinate and geometric grid for all of the BCD frames of a field and then equalized the background in overlapping frames. Unlike the mosaicking for the IRAC images, however, the $24 \mu \mathrm{m}$ BCDs did not require drizzling since the PSF is nearly Nyquist-sampled. Thus, we interpolated the BCDs into the common grid with a simple bilinear method, correcting the individual images for optical distortion. Deviant pixels (e.g., due to cosmic ray hits or bad array pixels) were identified and masked with the same method as used for the IRAC mosaics. The interpolated science and uncertainty images were finally co-added and combined into a single science and uncertainty mosaic, again weighting pixels by exposure time. The final mosaics have 1 1".275 pixels.

For the GOODS-N field, the reduced and mosaicked $24 \mu \mathrm{m}$ images were downloaded from the GOODS Spitzer Legacy Project's Web site. The processing of this data is described in R. Chary et al. (2009, in preparation). The final mosaic has 1 1".2 pixels, and its astrometry has been tied to the HST-ACS images of the GOODS-N field through the IRAC data of the same field.

\section{B.2. $24 \mu \mathrm{m}$ Source Extraction}

The high source density at $24 \mu \mathrm{m}$ in deep images (Papovich et al. 2004) combined with the large point response function (PRF) of MIPS (FWHM $=5^{\prime \prime} .9$ at $24 \mu \mathrm{m}$ ) causes significant source crowding in deep MIPS- $24 \mu \mathrm{m}$ images, especially as the image depth increases. Also, a significant fraction of a point-source's flux at $24 \mu \mathrm{m}(\sim 50 \%)$ is found in the Airy rings. As a result, reliable photometry measurements require flux profile fitting for each source. We used the APEX package included within MOPEX in single-frame mode to perform source detection and extraction through PRF fitting for all of the $24 \mu \mathrm{m}$ mosaics of the SMG fields, including GOODS-N.

An empirical PRF for each mosaic was created in a multi-step, iterative process, based on that described in Fadda et al. (2006). We first used a theoretical PRF generated from STinyTim (Krist 2002) to extract several tens of bright, relatively uncrowded point sources (no neighbors within $20^{\prime \prime}$ ) from which a preliminary empirical PRF was constructed. This empirical PRF was subsequently refined by subtracting all neighbors within $36^{\prime \prime}$ of the sources used to determine the PRF. This final PRF was normalized to the flux contained within a radius of 12 mosaic pixels (15.'3). We used the PRF constructed for each $24 \mu \mathrm{m}$ mosaic image to fit and extract of all point sources within that particular mosaic with $\mathrm{S} / \mathrm{N}>3$. An aperture correction of 1.153 was applied to the extracted fluxes to account for the normalization of 
the PRF flux within a 15" radius; this correction was derived by Fadda et al. (2006) from a curve-of-growth analysis of a bright star in the $24 \mu \mathrm{m}$ mosaic from the Spitzer First Look Survey (FLS) and is likely to be correct for our data set as the image processing, mosaicking, and source extraction procedures are similar.

For nondetections at $24 \mu \mathrm{m}$, we list a $3 \sigma$ upper limit on the point-source flux, which is calculated using the equation

$$
\sigma=\mathrm{RMS}_{\text {local }} \times N
$$

where $\mathrm{RMS}_{\text {local }}$ is the rms per pixel in a $20 \times 20$-pixel box centered on the radio position of the SMG in units of $\mu \mathrm{Jy}$ pixel $^{-1}$, and $N$ is the theoretical number of noise pixels ${ }^{17}$ in the $24 \mu \mathrm{m}$ mosaic.

The absolute fluxes measured in our source extraction process are likely to be accurate to better than $10 \%$ for bright sources $(\mathrm{S} / \mathrm{N}>20)$, including the uncertainty in the absolute flux calibration (4\%; Engelbracht et al. 2007) and uncertainties in the extraction process and aperture correction. For fainter sources $(5<\mathrm{S} / \mathrm{N}<10)$, there is likely an additional systematic $\sim 10 \%-15 \%$ uncertainty in the flux measurements introduced by the extraction procedure (Fadda et al. 2006). At low $\mathrm{S} / \mathrm{N}$ levels, extracted sources are biased toward higher fluxes since they are preferentially detected by APEX if they lie above positive fluctuations in the background noise. We do not include any flux uncertainty due to non-negligible color corrections needed to correct to monochromatic flux densities. For a power-law SED at $24 \mu \mathrm{m}$, such corrections would be less than 4\%; however, for $z \sim 1-3$ sources, redshifted emission and absorption features passing into the $24 \mu \mathrm{m}$ band (e.g., mid-IR PAH features, Menéndez-Delmestre et al. 2007) cause the color corrections to vary dramatically with redshift and mid-IR spectrum. Consequently, we have chosen not to apply systematic color corrections to the cataloged fluxes of sources extracted at $24 \mu \mathrm{m}$.

The absolute positions of extracted $24 \mu \mathrm{m}$ sources are expected to be accurate to within 1".5. Because we did not perform pointing refinement on the $24 \mu \mathrm{m}$ mosaics (apart from the GOODS-N mosaic, which was aligned to the VLA reference frame by Chary et al.) due to a lack of a significant number of bright 2MASS point-source counterparts at $24 \mu \mathrm{m}$, the uncertainties in the source positions are dominated by the blind pointing uncertainty of MIPS at $24 \mu \mathrm{m}\left(1 \sigma=1{ }^{\prime \prime} .2\right)$. Fadda et al. (2006) show through extractions of simulated sources that uncertainties resulting from the extraction process, dependent on $\mathrm{S} / \mathrm{N}$, also become important for sources with $\mathrm{S} / \mathrm{N}<10$. This extraction error, due to difficulty in centering the PRF for faint objects occupying relatively few pixels, is negligible for $\mathrm{S} / \mathrm{N}>20$, but can be as much as $\sim 1^{\prime \prime}$ for sources with $\mathrm{S} / \mathrm{N}<7$. Thus, position uncertainties for bright sources should be less than the stated 1 1".5, while $1^{\prime \prime} .5$ is generous enough to account for errors in the positions of low $\mathrm{S} / \mathrm{N}$ sources.

\section{B.3. Flux Comparison to Literature Measurements}

In the cases in which we have SMGs in common with the samples of Pope et al. (2006; 7), Egami et al. (2004; 4), and Ivison et al. $(2004 ; 3)$, which are detected at $24 \mu \mathrm{m}$, we have compared our flux measurements to those of the other authors. We find that our flux measurements agree to within $10 \%$ with those of Pope et al. (2006) and to within 12\% with those of

\footnotetext{
17 The number of noise pixels is the equivalent number of pixels whose noise contributes to the flux of an extracted point source.
}

Egami et al. (2004) and Ivison et al. (2004) with the exception of one source, SMM J105155.47, which has low S/N and is thus has a larger uncertainty in its flux (see Section B.2). However, the separate measurements of this SMG agree within their stated errors. When we compare our flux measurements of SMGs in the Lockman Hole to those of Ivison et al. (2007), however, agreement is more variable. Half of the sources have measurements which agree to within 5\%-10\% between the two studies, whereas others have fluxes which differ by $20 \%-50 \%$. Finally, as a check of our source extraction procedure in general, we have also compared our $24 \mu \mathrm{m}$ field source catalog from the entire GOODS-N field to that of R. Chary et al. (2009, in preparation), and find that our photometry agrees with that of Chary et al.- typically within $10 \%$.

\section{APPENDIX C MIPS-70 $\mu$ m DATA PROCESSING}

Processing of the $70 \mu \mathrm{m}$ MIPS data for all SMG fields followed the procedures described in Frayer et al. (2006a). The basic data reduction pipeline run by the SSC calculates the slope of the data ramps in each pixel, rejects cosmic rays, corrects nonlinearities, and calibrates the slope image by performing dark subtraction, flat-fielding, and absolute flux calibration. The pipeline also filters the $70 \mu \mathrm{m}$ images in the spatial and time domains, which improves the sensitivity of the $\mathrm{BCD}$ images by a factor of 2; however, the filtered images are only appropriate for point sources because information on the extended background is lost.

Because none of the SMG fields contain bright or extended sources at $70 \mu \mathrm{m}$, and in fact do not contain many sources at all, we began our reduction of all fields except the GOODS-N field with the filtered $70 \mu \mathrm{m}$ BCD images produced by v14.4 of the SSC MIPS data pipeline. The filtered BCDs for the primary array were next combined into a single-image mosaic for each SMG field using MOPEX. The individual filtered BCD frames were corrected for distortion and interpolated to a common sky grid in a similar way to the $24 \mu \mathrm{m}$ images. Bad array pixels were identified through temporal filtering only and masked prior to image co-addition. The reduction of the GOODS-N $70 \mu \mathrm{m}$ data is described in Frayer et al. (2006b).

All of the final $70 \mu \mathrm{m}$ image mosaics have square $4 "$ pixels.

\section{C.1. $70 \mu \mathrm{m}$ Source Extraction}

Frayer et al. (2006a) find that fitting a PRF to $70 \mu \mathrm{m}$ sources to obtain flux measurements provides more reliable results than aperture photometry, especially for faint sources. Thus, we used flux profile fitting to detect and extract fluxes for $70 \mu \mathrm{m}$ sources in the mosaics of the SMG fields, even though the source density in the $70 \mu \mathrm{m}$ images is significantly lower than in the $24 \mu \mathrm{m}$ images. We again used APEX to carry out PRF fitting on the $70 \mu \mathrm{m}$ images of all the SMG fields. However, due to a lack of bright sources in the images, we could not construct a PRF from our data. Instead, we used an empirical PRF constructed by Frayer et al. (2006a) from $70 \mu \mathrm{m}$ point sources detected in the xFLS to extract all $\mathrm{S} / \mathrm{N}>3$ sources in the SMG fields. The resulting flux densities were multiplied by a color correction factor of 1.09, which is appropriate for the typical range of IR SEDs for galaxies.

The absolute source fluxes extracted in the above procedure are expected to be accurate to $\sim 10 \%$, including the uncertainty in the absolute flux conversion factor (7\%; Gordon et al. 2007) and uncertainties in color corrections. The absolute positions of detected sources are expected to have an accuracy of $\sim 4^{\prime \prime}$, 
including the blind pointing uncertainty of MIPS at $70 \mu \mathrm{m} \mathrm{(1".7;}$ MIPS Data Handbook 2006) and uncertainty in the positions of low S/N extracted sources ( $\sim 3^{\prime \prime}$; Frayer et al. 2006a).

For SMGs not detected at $70 \mu \mathrm{m}$, we list the $3 \sigma$ upper limit on point-source flux as an upper limit. To calculate the $70 \mu \mathrm{m}$ pointsource flux upper limit, we use the empirical determination of the conversion factor between point-source noise and pixel surface brightness noise for 4" pixels from Frayer et al. (2006b) and the local rms per pixel for each object in a box centered on the radio position of the SMG:

$$
\sigma=\mathrm{RMS}_{\text {local }} \times 10.9 \mathrm{mJy}\left(\mathrm{MJy} \mathrm{sr}^{-1}\right)^{-1} .
$$

We have one source in common with Huynh et al. (2007), SMM J123634.51, for which our measurements are identical.

\section{REFERENCES}

Alexander, D. M., Bauer, F. E., Chapman, S. C., Smail, I., Blain, A. W., Brandt W. N., \& Ivison, R. J. 2005a, ApJ, 632, 736

Alexander, D. M., Smail, I., Bauer, F. E., Chapman, S. C., Blain, A. W., Brandt, W. N., \& Ivison, R. J. 2005b, Nature, 434, 738

Alexander, D. M., et al. 2003, AJ, 125, 383

Antonucci, R. 1993, ARA\&A, 31, 473

Appleton, P. N., et al. 2004, ApJS, 154, 147

Ashby, M. L. N., et al. 2006, ApJ, 644, 778

Barger, A. J., Cowie, L. L., Mushotzky, R. F., \& Richards, E. A. 2001, AJ, 121, 662

Barger, A. J., Cowie, L. L., \& Richards, E. A. 2000, AJ, 119, 2092

Barger, A. J., Cowie, L. L., \& Sanders, D. B. 1999a, ApJ, 518, L5

Barger, A. J., Cowie, L. L., Smail, I., Ivison, R. J., Blain, A. W., \& Kneib, J.-P. 1999b, AJ, 117, 2656

Barger, A. J., et al. 1998, Nature, 394, 428

Baugh, C. M., et al. 2005, MNRAS, 356, 1191

Baugh, C. M., et al. 2007, in ASP Conf. Ser. 375, From Z-Machines to ALMA: (Sub)Millimeter Spectroscopy of Galaxies, ed. A. J. Baker et al. (San Francisco, CA: ASP), 7

Bertin, E., \& Arnouts, S. 1996, A\&AS, 117, 393

Biggs, A. D., \& Ivison, R. J. 2008, MNRAS, 385, 893

Blain, A. W., Chapman, S. C., Smail, I., \& Ivison, R. 2004, ApJ, 611, 52

Borys, C., Scott, D., Chapman, S. C., Halpern, M., Nandra, K., \& Pope, A. 2004, MNRAS, 355, 485

Borys, C., Smail, I., Chapman, S. C., Blain, A. W., Alexander, D. M., \& Ivison, R. J. 2005, ApJ, 635, 853

Calzetti, D., Armus, L., Bohlin, R. C., Kinney, A. L., Koornneef, J., \& StorchiBergmann, T. 2000, ApJ, 533, 682

Chapman, S. C., Blain, A. W., Smail, I., \& Ivison, R. J. 2005, ApJ, 622, 772

Chapman, S. C., Lewis, G. F., Scott, D., Borys, C., \& Richards, E. 2002, ApJ, 570,557

Chapman, S. C., Richards, E. A., Lewis, G. F., Wilson, G., \& Barger, A. J. 2001, ApJ, 548, L147

Chapman, S. C., Smail, I., Windhorst, R., Muxlow, T., \& Ivison, R. J. 2004, ApJ, 611,732

Condon, J. J. 1992, ARA\&A, 30, 575

Daddi, E., et al. 2004, ApJ, 600, L127

Downes, A. J. B., Peacock, J. A., Savage, A., \& Carrie, D. R. 1986, MNRAS 218,31

Dye, S., et al. 2008, MNRAS, 386, 1107

Eales, S. A., Bertoldi, F., Ivison, R., Carilli, C., Dunne, L., \& Owen, F. 2003, MNRAS, 344, 169

Eales, S. A., et al. 1999, ApJ, 515, 518

Eales, S. A., et al. 2000, AJ, 120, 2244

Egami, E., et al. 2004, ApJS, 154, 130

Engelbracht, C. W., et al. 2007, PASP, 119, 994

Fadda, D., et al. 2006, AJ, 131, 2859

Franx, M., et al. 2003, ApJ, 587, L79
Frayer, D. T., Reddy, N. A., Armus, L., Blain, A. W., Scoville, N. Z., \& Smail, I. 2004a, AJ, 127, 728

Frayer, D. T., et al. 2004b, ApJS, 154, 137

Frayer, D. T., et al. 2006a, AJ, 131, 250

Frayer, D. T., et al. 2006b, ApJ, 647, L9

Gordon, K. D., et al. 2005, PASP, 117, 503

Gordon, K. D., et al. 2007, PASP, 119, 1019

Greve, T. R., et al. 2005, MNRAS, 359, 1165

Holland, W. S., et al. 1999, MNRAS, 303, 659

Huang, J.-S., et al. 2005, ApJ, 634, 137

Hughes, D., et al. 1998, Nature, 394, 241

Huynh, M. T., Pope, A., Frayer, D. T., \& Scott, D. 2007, ApJ, 659, 305

Ivison, R. J., et al. 1998, MNRAS, 298, 583

Ivison, R. J., et al. 2002, MNRAS, 337, 1

Ivison, R. J., et al. 2004, ApJS, 154, 124

Ivison, R. J., et al. 2007, MNRAS, 380, 199

Joseph, R. D., Meikle, W. P. S., Robertson, N. A., \& Wright, G. S. 1984, MNRAS, 209, 111

Kovács, A., Chapman, S. C., Dowell, C. D., Blain, A. W., \& Phillips, T. G. 2006, ApJ, 650, 592

Krist, J. 2002, Tiny Tim/SIRTF Users Guide (Pasadena, CA: SSC)

Lacy, M., et al. 2004, ApJS, 154, 166

Lacy, M., et al. 2007, AJ, 133, 186

Lilly, S., et al. 1999, ApJ, 518, 641

Makovoz, D., \& Marleau, F. R. 2005, PASP, 117, 1113

Menéndez-Delmestre, K., et al. 2007, ApJ, 655, L65

Menéndez-Delmestre, K., et al. 2009, ApJ, in press

Neri, R., et al. 2003, ApJ, 597, L113

Osterbrock, D. E. 1989, Astrophysics of Gaseous Nebulae and Active Galactic Nuclei (Sausalito: University Science Books)

Papovich, C., et al. 2004, ApJS, 154, 70

Papovich, C., et al. 2006, ApJ, 640, 92

Pope, A., et al. 2006, MNRAS, 370, 1185

Pope, A., et al. 2008, ApJ, 675, 1171

Reddy, N. A., Steidel, C. C., Erb, D. K., Shapley, A. E., \& Pettini, M. 2006b, ApJ, 653, 1004

Sajina, A., Lacy, M., \& Scott, D. 2005, ApJ, 621, 256

Sajina, A., Yan, L., Armus, L., Choi, P., Fadda, D., Helou, G., \& Spoon, H. 2007a, ApJ, 664, 713

Sajina, A., Yan, L., Lacy, M., \& Huynh, M. 2007b, ApJ, 667, L17

Scott, S. E., et al. 2002, MNRAS, 331, 817

Scoville, N. Z., et al. 2000, AJ, 119, 991

Seymour, N., et al. 2007, ApJS, 171, 353

Seymour, N., et al. 2008, ApJ, 681, L1

Siebenmorgen, R., \& Krügel, E. 2007, A\&A, 461, 445

Smail, I., Chapman, S. C., Blain, A. W., \& Ivison, R. J. 2004, ApJ, 616, 71

Smail, I., Ivison, R. J., \& Blain, A. W. 1997, ApJ, 490, L5

Smail, I., Ivison, R. J., Blain, A. W., \& Kneib, J.-P. 1998, ApJ, 507, L21

Smail, I., Ivison, R. J., Blain, A. W., \& Kneib, J.-P. 2002, MNRAS, 331, 495

Smail, I., Ivison, R. J., Owen, F. N., Blain, A. W., \& Kneib, J.-P. 2000, ApJ, 528,612

Spinoglio, L., Malkan, M. A., Rush, B., Carrasco, L., \& Recillas-Cruz, E. 1995, ApJ, 453, 616

Spitzer Science Center. 2006, MIPS Data Handbook (Pasadena, CA: SSC), http://ssc.spitzer.caltech.edu/mips/dh/

Steidel, C. C., Adelberger, K. L., Shapley, A. E., Pettini, M., Dickinson, M., \& Giavalisco, M. 2003, ApJ, 592, 728

Stern, D., et al. 2005, ApJ, 631, 163

Swinbank, A. M., Smail, I., Chapman, S. C., Blain, A. W., Ivison, R. J., \& Keel, W. C. 2004, ApJ, 617, 64

Swinbank, A. M., et al. 2008, MNRAS, 391, 420

Tacconi, L. J., et al. 2006, ApJ, 640, 228

Tacconi, L. J., et al. 2008, ApJ, 680, 246

Takata, T., et al. 2006, ApJ, 651, 713

Valiante, E., Lutz, D., Sturm, E., Genzel, R., Tacconi, L. J., Lehnert, M. D., \& Baker, A. J. 2007, ApJ, 660, 1060

Webb, T. M., et al. 2003, ApJ, 587, 41

Weedman, D., et al. 2006, ApJ, 653, 101

Yan, L., et al. 2007, ApJ, 658, 778

Yun, M. S., et al. 2008, MNRAS, 389, 333 